\title{
STRONG COMPLETENESS OF S4 FOR ANY DENSE-IN-ITSELF METRIC SPACE
}

\author{
PHILIP KREMER
}

Department of Philosophy, University of Toronto

\begin{abstract}
In the topological semantics for modal logic, S4 is well-known to be complete for the rational line, for the real line, and for Cantor space: these are special cases of S4's completeness for any dense-in-itself metric space. The construction used to prove completeness can be slightly amended to show that S4 is not only complete, but also strongly complete, for the rational line. But no similarly easy amendment is available for the real line or for Cantor space and the question of strong completeness for these spaces has remained open, together with the more general question of strong completeness for any dense-in-itself metric space. In this paper, we prove that S4 is strongly complete for any dense-in-itself metric space.
\end{abstract}

In the topological semantics for modal logic (McKinsey, 1941; McKinsey \& Tarski, 1944; Rasiowa \& Sikorski, 1963), S4 is well-known to be complete for the class of all topological spaces, as well as for a number of particular topological spaces, notably the rational line, $\mathbb{Q}$, the real line, $\mathbb{R}$, and Cantor space, $\mathcal{C}$. The results for $\mathbb{Q}, \mathbb{R}$, and $\mathcal{C}$ are special cases of S4's completeness for any dense-in-itself metric space: see Rasiowa \& Sikorski (1963, Theorem XI, 9.1), which is derived from McKinsey (1941) and McKinsey \& Tarski (1944).

In Kripke semantics, it is customary to strengthen the completeness of a logic, L, for a Kripke frame (a class of frames) to strong completeness, that is, the claim that any L-consistent set of formulas is satisfiable at some point in the frame (in some frame in the class): the simplest completeness arguments, using canonical frames and models, tend to deliver this stronger result. ${ }^{1}$ In topological semantics, as long as the language is countable, the construction used to prove the completeness of $S 4$ for $\mathbb{Q}$ can be slightly amended to show the strong completeness of S4 for $\mathbb{Q}$. But no similarly easy amendment is available for $\mathbb{R}$ or for $\mathcal{C}$, and the question of the strong completeness of $\mathrm{S} 4$ for these spaces has until now remained open. In the current paper, we prove that $\mathrm{S} 4$ is strongly complete for any dense-in-itself metric space, including $\mathbb{R}$ and $\mathcal{C}$.

It should be noted that the question of strong completeness in topological semantics has not been widely addressed. That said, given the well-known identification of reflexive transitive Kripke frames with Alexandroff spaces (see p. 549, below), and given the wellknown strong completeness of S4 for the class of countable reflexive transitive Kripke frames (Makinson, 1966), S4 is strongly complete for the class of countable Alexandroff spaces and therefore for the class of all topological spaces. Also, Theorem XI, 10.2 (v) in Rasiowa \& Sikorski (1963) immediately entails a kind of restricted strong completeness of

Received: February 26, 2013.

1 Such arguments were first published in Makinson (1966). There are, however, modal logics L that are complete for some class of Kripke frames for L, but strongly complete for no class of Kripke frames for L. Standard examples are GL and Grz: see Zakharyaschev et al. (1997), especially Section 1.5, "Stronger forms of Kripke completeness". 
S4 for the class of subspaces of the irrational line: any consistent theory is satisfiable in this class, where a theory is a set of formulas containing all the theorems of S4 and closed under both modus ponens and necessitation. These results leave open the strong completeness of $\mathrm{S} 4$ for $\mathbb{R}$, for $\mathcal{C}$, and for any arbitrary dense-in-itself metric space.

\section{§1. Outline.}

1.1. An interior map strategy. The completeness of $\mathrm{S} 4$ for a given dense-in-itself metric space $X$ is typically proved by showing that any finite rooted reflexive transitive Kripke frame is the image of an interior map from $X$. Section $\S 3$, below, strengthens the completeness of $\mathrm{S} 4$ for $\mathbb{Q}$ to strong completeness by showing that any countable rooted reflexive transitive Kripke frame is the image of an interior map from $\mathbb{Q}$. We pursue this strategy in two steps. Firstly, we observe that any countable rooted reflexive transitive Kripke frame can be unravelled into the infinite binary tree, $2^{<\omega}$ (Lemma 3.3): thus S4 is strongly complete for $2^{<\omega}$ (Lemma 3.4). Secondly, we observe that there is an interior map from $\mathbb{Q}$ onto $2^{<\omega}$ : thus $\mathrm{S} 4$ is strongly complete for $\mathbb{Q}$. This is an instance of a general strategy. First, show that $\mathrm{S} 4$ is strongly complete for some master space $Y$, in this case $2^{<\omega}$. Then transfer the strong completeness of $\mathrm{S} 4$ for the master space to the strong completeness of $\mathrm{S} 4$ for $X$, backwards via a surjective interior map, as in Figure 1.

The interior map strategy just outlined shows the strong completeness of S4 for a particular space by showing that every countable rooted reflexive transitive Kripke frame is the image of that space under some interior map. But this strategy is not applicable to every dense-in-itself metric space: for example, the Kripke frame $\langle\mathbb{N}, \leq\rangle$ is not the image of any interior map from $\mathbb{R}$-see Lemma 4.6. ${ }^{2}$ Neither is $2^{<\omega}$-see Corollary 4.8. This observation has motivated the strong suspicion that there is a set $\Gamma$ of formulas satisfiable in $\langle\mathbb{N}, \leq\rangle$, and therefore consistent, but not satisfiable in $\mathbb{R}$. This suspicion is refuted by our main result. Section $\$ 4$ also considers another interior map strategy and shows that it too fails.

1.2. Algebraic semantics. Sections $\S 5 \mathrm{ff}$ are devoted to the main project: proving that S4 is strongly complete for any dense-in-itself metric space. Section $\$ 5$ generalizes the topological semantics for $\mathrm{S} 4$ to an algebraic semantics. Any topological space $X$ generates an interior algebra $\mathcal{I}(X)=\left\langle\mathcal{P}(X), \subseteq, I n t_{X}\right\rangle$, where $\operatorname{Int}_{X}$ is the interior operator on subsets of $X$. In general, an interior algebra is a triple $\mathcal{I}=\langle\mathbf{A}, \leq, \mathbf{I}\rangle$, where $\langle\mathbf{A}, \leq\rangle$ is a Boolean algebra, and $\mathbf{I}$ is a unary interior function on $\mathbf{A}$ satisfying certain conditions. ${ }^{3}$ Section $\$ 5$ generalizes topological models to algebraic models based on interior algebras and defines what it is for $\mathrm{S} 4$ to be strongly complete for an interior algebra $\mathcal{I}$ : the strong completeness of $\mathrm{S} 4$ for the topological space $X$ is then equivalent to the strong completeness of $\mathrm{S} 4$ for the interior algebra $\mathcal{I}(X)$. So, to prove the strong completeness $\mathrm{S} 4$ for any dense-in-itself metric space $X$, it suffices to transfer the strong completeness of $\mathrm{S} 4$ from $\mathcal{I}\left(2^{<\omega}\right)$ to $\mathcal{I}(X)$.

With topological spaces, the strong completeness of S4 is transferred backwards from the range of a surjective interior map onto the domain as in Figure 1. With interior algebras, the strong completeness of S4 is transferred forwards from the domain of an embedding to

2 I owe this observation to Guram Bezhanishvili, David Gabelaia, and Valentin Shehtman.

3 These conditions are the duals of the Kuratowski axioms for a closure function, introduced in Kuratowski (1922). See Section §5, below. 


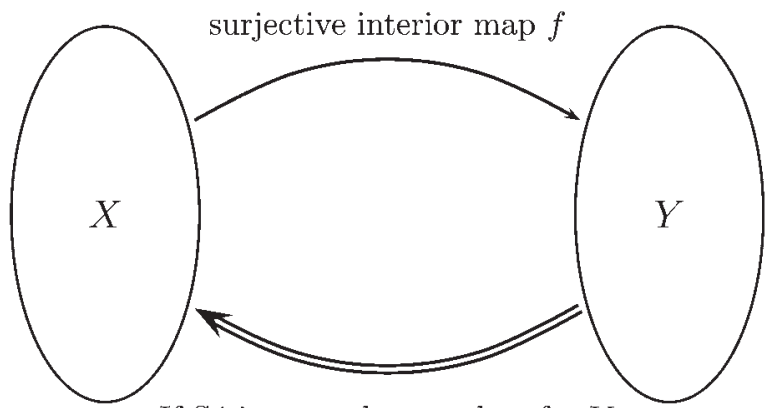

If $\mathrm{S} 4$ is strongly complete for $Y$

then $\mathrm{S} 4$ is strongly complete for $X$.

Fig. 1. Transferring strong completeness from one topological space to another, backwards via a surjective interior map.

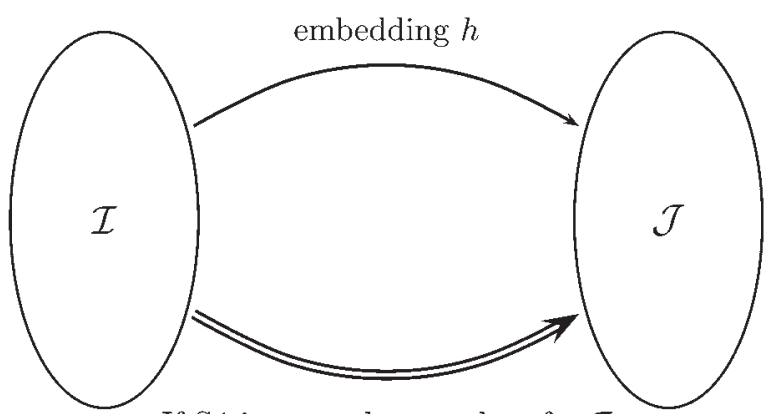

If $\mathrm{S} 4$ is strongly complete for $\mathcal{I}$

then $\mathrm{S} 4$ is strongly complete for $\mathcal{J}$.

Fig. 2. Transferring the strong completeness of S4 from one interior algebra to another, forwards via an embedding.

the range as in Figure 2. So our task reduces to finding an embedding from $\mathcal{I}\left(2^{<\omega}\right)$ into $\mathcal{I}(X)$.

1.3. Strong completeness for the infinite binary tree with limits. Section $\S 6$ considers the infinite binary tree with limits: the space $2^{\leq \omega}$ of finite and infinite binary sequences, equipped with the Scott topology. ${ }^{4}$ We define an embedding, $h_{\mathcal{U}}$ (the reason for the subscripted $\mathcal{U}$ will become clearer below), from $\mathcal{I}\left(2^{<\omega}\right)$ into $\mathcal{I}\left(2^{\leq \omega}\right)$, thereby transferring the strong completeness of S4 from $\mathcal{I}\left(2^{<\omega}\right)$ to $\mathcal{I}\left(2^{\leq \omega}\right)$ - and so transferring the strong completeness of $\mathrm{S} 4$ from $2^{<\omega}$ to $2^{\leq \omega}$. 5,6 Note that the the embedding $h_{\mathcal{U}}$ cannot be onto, since $\mathcal{P}\left(2^{\leq \omega}\right)$ is of greater cardinality than $\mathcal{P}\left(2^{<\omega}\right)$. If we let the algebra $\mathcal{J}_{\mathcal{U}}$ be the range of $h_{\mathcal{U}}$, then note: (1) $\mathcal{J}_{\mathcal{U}}$ is a proper subalgebra of $\mathcal{I}\left(2^{\leq \omega}\right)$, and (2) $h_{\mathcal{U}}$ transfers the strong completeness of S4 from $\mathcal{I}\left(2^{<\omega}\right)$ to $\mathcal{J}$. This will be useful below.

4 Nick Bezhanishvili pointed out that the topology used in Section $\$ 6$ is the Scott topology.

5 In fact, we define a whole class of embeddings from $\mathcal{I}\left(2^{<\omega}\right)$ into $\mathcal{I}\left(2^{\leq \omega}\right)$, though we only need one of them.

6 An anonymous referee alerted me to Lando \& Sarenac (2011), which proves that S4 is complete for $2 \leq \omega$. The interior map strategy used in Lando \& Sarenac (2011) does not extend to proving strong completeness: see Section $§ 6$, below. 


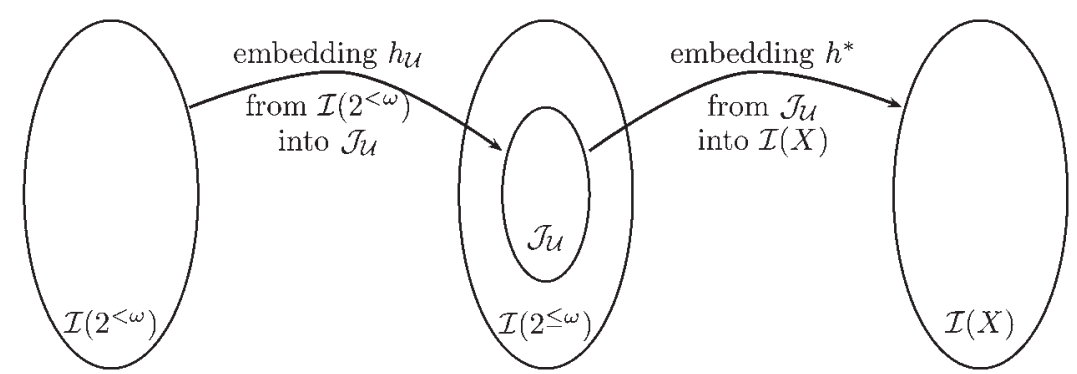

Fig. 3. Strong completeness is transferred from $\mathcal{I}\left(2^{<\omega}\right)$ to $\mathcal{I}(X)$ via the embedding $h^{*} \circ h_{\mathcal{U}}$.

1.4. Strong completeness for dense-in-themselves metric spaces. Section $\S 7$ defines, for any dense-in-itself metric space $X$, a function $f_{X}: X \rightarrow 2^{\leq \omega}$ and shows that this function is continuous. Section $\S 8$ shows that, if $X$ is a complete dense-in-itself metric space, then $f_{X}$ is a surjective interior map. ${ }^{7}$ Thus, when $X$ is a complete dense-in-itself metric space, we can transfer the strong completeness of $\mathrm{S} 4$ from $2^{\leq \omega}$ to $X$ backwards via $f_{X}$. An aside, the existence of an interior map from any complete dense-in-itself metric space onto $2^{\leq \omega}$ might be of broader interest, for example, in studying logics of the real line and of other complete dense-in-themselves metric spaces in other contexts, such as dynamic topological logic (see Kremer \& Mints, 2005) or bimodal logic in twodimensional topological semantics (see van Benthem et al., 2006; Kremer, xxxx).

Unfortunately, there are incomplete dense-in-themselves metric spaces $X$ such that the function $f_{X}: X \rightarrow 2^{\leq \omega}$ is neither an interior map nor surjective. In such cases, we cannot simply transfer the strong completeness of $\mathrm{S} 4$ from $2^{\leq \omega}$ to $X$ backwards via $f_{X}$. Fortunately, even in such cases, the function $f_{X}$ induces an embedding, say $h^{*}$, from the subalgebra $\mathcal{J}_{\mathcal{U}}$ of $\mathcal{I}\left(2^{\leq \omega}\right)$ into $\mathcal{I}(X)$. So the strong completeness of S4 is transferred first from $\mathcal{I}\left(2^{\leq \omega}\right)$ to $\mathcal{J}_{\mathcal{U}}$ via the embedding $h_{\mathcal{U}}$, and then from $\mathcal{J}_{\mathcal{U}}$ to $\mathcal{I}(X)$ via the embedding $h^{*}$ : see Figure 3. Note that $h^{*} \circ h_{\mathcal{U}}$ is an embedding from $\mathcal{I}\left(2^{<\omega}\right)$ into $\mathcal{I}(X)$. This suffices for the strong completeness of S4 for $X$.

\$2. Notation, terminology, and main result. We begin by fixing notation and terminology. We assume a propositional language with a countable set $P V$ of propositional variables; standard Boolean connectives $\&, \vee$, and $\neg$; and one modal operator, $\square$. We abbreviate $\neg \square \neg A$ as $\diamond A$ and $(\neg A \vee B)$ as $(A \supset B)$. A finite set of formulas is consistent iff either it is empty or the negation of the conjunction of the formulas in it is not a theorem of S4; and an infinite set of formulas is consistent iff every finite subset is consistent.

Given a nonempty set $X$, a topology on $X$ is a family $\tau$ of subsets of $X$, such that (1) $\emptyset, X \in \tau$; (2) if $S, S^{\prime} \in \tau$, then $S \cap S^{\prime} \in \tau$; and (3) if $\sigma \subseteq \tau$, then $\bigcup \sigma \in \tau$. Dugundji (1966) and Engelking (1989) are standard references on topology. The members of $\tau$ are the open subsets of $X$ (in the topology $\tau$ ). A basis for $\tau$ is any set $\sigma \subseteq \tau$ such that every member of $\tau$ is the union of members of $\sigma$. A topological space is an ordered pair $\langle X, \tau\rangle$,

7 After I showed him the construction of an interior map from $\mathbb{R}$ onto $2^{\leq \omega}$, David Gabelaia conjectured that the construction could be generalized to any complete dense-in-itself metric space. He was right. An anonymous referee has alerted me to Lando (2012), which constructs an independently discovered interior map from $\mathbb{R}$ onto $2^{\leq \omega}$. 
where $X$ is a nonempty set and $\tau$ is a topology on $X$. We will somewhat imprecisely identify $X$ with $\langle X, \tau\rangle$, letting context or fiat determine $\tau$. Thus, for example, we identify $\mathbb{R}$ with $\left\langle\mathbb{R}, \tau_{\mathbb{R}}\right\rangle$, where $\tau_{\mathbb{R}}$ is the standard topology on $\mathbb{R}$. We take the basics of pointset topology to be given, in particular, the notions of the interior and closure, $\operatorname{Int}_{X}(S)$ and $C l_{X}(S)$, of a subset $S$ of a topological space, $X$. The boundary of a set $S \subseteq X$ is $\partial_{X}(S)={ }_{\mathrm{df}} C l_{X}(S)-\operatorname{Int}_{X}(S)$. We typically suppress the subscripted $X$ on $\operatorname{Int}_{X}, C l_{X}$, and $\partial_{X}$. A subset $S$ of $X$ is dense iff $X=C l(S)$. A point $x \in X$ is an isolated point iff $\{x\}$ is open. A space $X$ is dense-in-itself iff $X$ has no isolated points. Alternatively, $X$ is dense-in-itself iff $(\forall x \in X)(C l(X-\{x\})=X)$.

A Kripke frame is an ordered pair $\langle X, R\rangle$, where $X$ is a nonempty set and $R \subseteq X \times X$. We will somewhat imprecisely identify $X$ with $\langle X, R\rangle$, letting context or fiat determine $R$. A Kripke frame $X$ is reflexive (transitive) iff $R$ is: for the rest of this paper, we will assume that all Kripke frames are reflexive and transitive. A Kripke frame is rooted iff $(\exists r \in X)(\forall x \in X)(r R x)$. A subset $O$ of $X$ is open iff $(\forall x, y \in X)(x \in O \& x R y \Rightarrow$ $y \in O)$. A subset $C$ of $X$ is closed iff $X-C$ is open. If $\langle X, R\rangle$, is a Kripke frame, then the open sets just defined form a topology, $\tau_{R}$ on $X$. Indeed, the topological space $\left\langle X, \tau_{R}\right\rangle$ is an Alexandroff space: these are the spaces $\langle X, \tau\rangle$ such that, if $\sigma \subseteq \tau$, then $\bigcap \sigma \in \tau$. We will somewhat imprecisely identify the Kripke frame $\langle X, R\rangle$ with the Alexandroff space $\left\langle X, \tau_{R}\right\rangle$. If $\langle X, R\rangle$ is a Kripke frame, then the interior of a set $S \subseteq X$ is the largest open subset of $S: \operatorname{Int}_{X}(S)={ }_{\mathrm{df}}\{x \in S: \forall y \in X, x R y \Rightarrow y \in S\}$. Finally, for $x \in X$, we define $R(x)=_{\mathrm{df}}\{y \in X: x R y\}$. Note that the family $\{R(x): x \in X\}$ is a basis for the topology $\tau_{R}$.

A topological model (Kripke model) is an ordered pair $\mathbf{M}=\langle X, V\rangle$, where $X$ is a topological space (Kripke frame) and $V: P V \rightarrow \mathcal{P}(X) . V$ is called a valuation. We will use the term model to cover topological models and Kripke models. For any model $\mathbf{M}=\langle X, V\rangle, V$ is extended to all formulas as follows: $V(\neg A)=X-V(A), V(A \& B)=$ $V(A) \cap V(B), V(A \vee B)=V(A) \cup V(B)$, and $V(\square A)=\operatorname{Int}(V(A))$. If $\Gamma$ is a nonempty set of formulas, then $V(\Gamma)=_{\mathrm{df}} \bigcap_{A \in \Gamma} V(A)$; if $\Gamma$ is empty, then $V(\Gamma)=_{\mathrm{df}} X$.

Suppose that $A$ is a formula, that $X$ is a topological space or Kripke frame, and that $\mathbf{M}=\langle X, V\rangle$ is a model. We say that $A$ is valid in $\mathbf{M}$ iff $V(A)=X$, and valid in $X$ iff $A$ is valid in $\langle X, V\rangle$ for every valuation $V$. We say that $\mathrm{S} 4$ is complete for $X$ iff $A \in \mathrm{S} 4$ for every formula $A$ valid in $X$.

Suppose that $\Gamma$ is a set of formulas. Suppose that $X$ is a topological space or Kripke frame, that $x \in X$, and that $V: P V \rightarrow \mathcal{P}(X)$ is a valuation. Then we say that $\Gamma$ is satisfied at $x$ in $\langle X, V\rangle$ iff $x \in V(\Gamma)$, that $\Gamma$ is satisfiable at $x$ in $X$ iff there is some model $\langle X, V\rangle$ such that $x \in V(\Gamma)$, and that $\Gamma$ is satisfiable in $X$ iff $\Gamma$ is satisfiable at some $x \in X$. Note that S4 is complete for $X$ iff every finite consistent set of formulas is satisfiable in $X$. We say that $\mathrm{S} 4$ is strongly complete for $X$ iff every consistent set of formulas is satisfiable in $X$.

A metric space is an ordered pair $\langle X, d\rangle$, where $X$ is a nonempty set and the distance function $d: X \times X \rightarrow \mathbb{R}$ satisfies the following for every $x, y, z \in X$ :

1. $d(x, y)=d(y, x) \geq 0$,

2. $d(x, y)=0$ iff $x=y$, and

3. $d(x, z) \leq d(x, y)+d(y, z)$ (the triangle inequality).

We will not carefully distinguish $X$ from $\langle X, d\rangle$. It will be useful to define the distance between a point $x \in X$ and a set $S \subseteq X$. If $S$ is nonempty, then

$$
d(x, S)=_{\mathrm{df}} \inf \{d(x, y): y \in S\} .
$$


And $d(x, \emptyset)={ }_{\mathrm{df}} \infty$. It will also be useful to note that a kind of triangle inequality holds for distances to nonempty sets as well as to points:

$$
d(x, S) \leq d(x, y)+d(y, S)
$$

We impose a topology $\tau_{d}$ on $X$ by taking as a basis all the open balls, that is, all the sets of the following form, where $x \in X$ and $r \in \mathbb{R}^{+}: \mathbf{B}(x, r)=_{\mathrm{df}}\{y: d(x, y)<r\}$-here, $\mathbb{R}^{+}$ is the set of positive reals. Note the following: if $O \subseteq X$ is open, then $(\forall x \in X)(x \in O$ iff $\left.\left(\exists r \in \mathbb{R}^{+}\right)(\mathbf{B}(x, r) \subseteq O)\right)$. We will not carefully distinguish $X$ and $\langle X, d\rangle$ from $\left\langle X, \tau_{d}\right\rangle$. Note that if we impose the distance function $d(x, y)=|x-y|$ on $\mathbb{R}, \mathbb{Q}$, and $\mathcal{C}$, then they are all dense-in-themselves metric spaces.

Suppose that $X$ is a metric space with distance function $d$, that $\left\langle x_{n}\right\rangle=x_{1}, \ldots, x_{n}, \ldots$ is an infinite sequence of points in $X$ and that $x \in X$. Then $\left\langle x_{n}\right\rangle$ is a Cauchy sequence iff $(\forall \varepsilon>0)(\exists n)(\forall i, j \geq n)\left(d\left(x_{i}, x_{j}\right)<\varepsilon\right)$. We say that $\left\langle x_{n}\right\rangle$ converges to $x$ iff $(\forall \varepsilon>$ $0)(\exists n)(\forall m \geq n)\left(d\left(x, x_{m}\right)<\varepsilon\right)$. We write $\left\langle x_{n}\right\rangle \rightarrow x$. We say that $\left\langle x_{n}\right\rangle$ converges iff $(\exists x \in X)\left(\left\langle x_{n}\right\rangle \rightarrow x\right)$. Note that every convergent sequence is Cauchy. Finally, a metric space is complete iff every Cauchy sequence converges. It is well-known that $\mathbb{R}$ and $\mathcal{C}$ are complete but that $\mathbb{Q}$ is not.

The following completeness theorem is taken from Rasiowa \& Sikorski (1963, Theorem XI, 9.1, (vii)), which is in turn derived from McKinsey (1941) and McKinsey \& Tarski (1944):

THEOREM 2.1. (Completeness). If X is a dense-in-itself metric space, then $\mathrm{S} 4$ is complete for $X$.

\section{Our main result is}

THEOREM 2.2. (Strong completeness). If $X$ is a dense-in-itself metric space, then $\mathrm{S} 4$ is strongly complete for $X$.

Theorem 2.1 is well-known, especially when $X=\mathbb{Q}, \mathbb{R}$, or $\mathcal{C}$. For $X=\mathbb{Q}$, there is a new and more accessible proof in van Benthem et al. (2006); and, for $X=\mathbb{R}$, there are new and more accessible proofs in Aiello et al. (2003), Bezhanishvili \& Gehrke (2005), Mints \& Zhang (2005) and Hodkinson (2012). For $X=\mathbb{Q}$, Theorem 2.2 is easy to prove and seems to be well-known (though we have not found an explicit statement of it in the literature): we sketch the easy proof in Section $\S 3$ below. For $X=\mathbb{R}$ or $\mathcal{C}$, and in the most general case, Theorem 2.2 has been an open question. As noted above, we devote Sections $\S 5 \mathrm{ff}$ to its proof.

We recall the standard notion of an interior map. A function from a topological space (or Kripke frame) to a topological space (or Kripke frame) is continuous iff the preimage of every open set is open; ${ }^{8}$ is open iff the image of every open set is open; ${ }^{9}$ and is an interior map iff it is continuous and open. Suppose that $\mathbf{M}=\langle X, V\rangle$ and $\mathbf{M}^{\prime}=\left\langle X^{\prime}, V^{\prime}\right\rangle$ are models, and that $f$ is an interior map from $X$ onto $X^{\prime}$. Then $f$ is an interior map from $\mathbf{M}$ onto $\mathbf{M}^{\prime}$ iff, for every $p \in P V$ and $x \in X, x \in V(p)$ iff $f(x) \in V^{\prime}(p)$. The following lemma and corollary are standard.

8 If $\langle X, R\rangle$ and $\left\langle X^{\prime}, R^{\prime}\right\rangle$ are Kripke frames, then $f: X \rightarrow X^{\prime}$ is a continuous function from the topological space $\left\langle X, \tau_{R}\right\rangle$ to the topological space $\left\langle X^{\prime}, \tau_{R^{\prime}}\right\rangle$ iff $f$ is monotonic: for every $x, y \in X$, if $x R y$ then $f x R^{\prime} f y$.

9 If $\langle X, R\rangle$ and $\left\langle X^{\prime}, R^{\prime}\right\rangle$ are Kripke frames, then $f: X \rightarrow X^{\prime}$ is an open function from the topological space $\left\langle X, \tau_{R}\right\rangle$ to the topological space $\left\langle X^{\prime}, \tau_{R^{\prime}}\right\rangle$ iff for every $x \in X$ and $y \in X^{\prime}$, if $f x R^{\prime} y$, then for some $z \in X, x R z$ and $f z=y$. 
LEMMA 2.3. If $f$ is an interior map from $\mathbf{M}=\langle X, V\rangle$ onto $\mathbf{M}^{\prime}=\left\langle X^{\prime}, V^{\prime}\right\rangle$, then for every formula $A$ and $x \in X, x \in V(A)$ iff $f(x) \in V^{\prime}(A)$.

COROLLARY 2.4. (Transfer of the strong completeness of S4 backwards via a surjective interior map). Suppose that each of $X$ and $X^{\prime}$ is a Kripke frame or topological space, and that there is an interior map from $X$ onto $X^{\prime}$. Then if $\Gamma$ is satisfiable in $X^{\prime}$ then $\Gamma$ is satisfiable in $X$. Thus, if S4 is strongly complete for $X^{\prime}$, then $\mathrm{S} 4$ is strongly complete for $X$.

§3. Strong completeness for the infinite binary tree and for the rational line: the interior map strategy. For each $n \geq 0$, let $2^{n}$ be the set of binary sequences (sequences of $0 \mathrm{~s}$ and $1 \mathrm{~s}$ ) of length $n$. Let $2^{<\omega}={ }_{\mathrm{df}} \bigcup_{n=0}^{\infty} 2^{n}$, that is, $2^{<\omega}$ is the set of finite binary sequences. Let $2^{\omega}$ be the set of infinite binary sequences of order type $\omega$. And let $2^{\leq \omega}={ }_{\mathrm{df}}$ $2^{<\omega} \cup 2^{\omega}$. We use $\Lambda$ for the the empty binary sequence, that is, the binary sequence of length 0 . We use $b, b^{\prime}$, etc., to range over $2^{<\omega} ; \mathbf{b}, \mathbf{b}^{\prime}$, etc., to range over $2^{\omega}$; and $\boldsymbol{b}, \boldsymbol{b}^{\prime}$, etc., to range over $2^{\leq \omega}$. If $b \in 2^{<\omega}$ and $\boldsymbol{b} \in 2^{\leq \omega}$, then we write $b^{\frown} \boldsymbol{b}$ for $b$ concatenated with $\boldsymbol{b}$. We write $b 0$ and $b 1$ for $b^{\frown}\langle 0\rangle$ and $b^{\frown}\langle 1\rangle$. For any $b \in 2^{<\omega}$, we write $|b|$ for the length of $b$. Given any $\mathbf{b} \in 2^{\omega}$ and any $n \in \mathbb{N}$, the finite binary sequence $\left.\mathbf{b}\right|_{n}$ is the initial segment of length $n$ of $\mathbf{b}$. Thus $\left.\mathbf{b}\right|_{0}=\Lambda$ and $|\mathbf{b}|_{n} \mid=n$. Given $b \in 2^{<\omega}$ and $\boldsymbol{b} \in 2^{\leq \omega}$, we say $b \leq \boldsymbol{b}$ iff $b$ is an initial segment of $\boldsymbol{b}$ and $b<\boldsymbol{b}$ iff both $b \leq \boldsymbol{b}$ and $b \neq \boldsymbol{b}$. We will also use " $\leq$ " for $\leq$ restricted to $2^{<\omega}$.

We identify $2^{<\omega}$ with the infinite binary tree, that is, the countably infinite rooted transitive reflexive Kripke frame $\left\langle 2^{<\omega}, \leq\right\rangle$. For each $b \in 2^{<\omega}$, let $[b]==_{\mathrm{df}}\left\{b^{\prime} \in 2^{<\omega}: b \leq b^{\prime}\right\}$. Note that the family $\left\{[b]: b \in 2^{<\omega}\right\}$ is a basis for the topology $\tau_{\leq}$on $2^{<\omega}$ induced by $\leq$. We also identify $2^{<\omega}$ with the topological space $\left\langle 2^{<\omega}, \tau_{\leq}\right\rangle$. We can represent any branch of the tree $2^{<\omega}$ with an infinite binary sequence $\mathbf{b} \in 2^{\omega}: \mathbf{b}$ represents the branch whose nodes are $\left.\mathbf{b}\right|_{0},\left.\mathbf{b}\right|_{1},\left.\mathbf{b}\right|_{2}, \ldots$. The following result, due originally to Dov Gabbay and independently discovered by Johan van Benthem, is well-known; for a proof see Goldblatt (1980, Theorem 1).

LEMMA 3.1. Any finite rooted reflexive transitive Kripke frame is the image of $2^{<\omega}$ under some interior map.

Together with the fact that any finite consistent set $\Gamma$ of formulas is satisfiable in some finite rooted reflexive transitive Kripke frame, Lemma 3.1 entails that S4 is complete for $2^{<\omega}$.

EXAMPLE 3.2. Here we give an example of a consistent set $\Gamma$ of sentence that is not satisfiable in any finite Kripke frame: this shows that Lemma 3.1 is not of immediate help for the strong completeness of $\mathrm{S} 4$ for $2^{<\omega}$. Suppose that $p_{0}, p_{1}, p_{2}, \ldots$ are distinct propositional variables. Let $\Gamma=\left\{\diamond p_{i}: i \in \mathbb{N}\right\} \cup\left\{\square\left(p_{i} \supset \neg p_{j}\right): i, j \in \mathbb{N}, i \neq j\right\}$. To see that $\Gamma$ is satisfiable, and therefore consistent, let $M=\langle\langle\mathbb{N}, \leq\rangle, V\rangle$, where $V\left(p_{n}\right)=\{n\}$. Note that $V(\Gamma)=\{0\}$. Thus, $\Gamma$ is satisfiable. But we also claim that $\Gamma$ is not satisfiable in any finite Kripke frame. For suppose that $\Gamma$ is satisfiable in the Kripke frame $\langle X, R\rangle$. Then there is some valuation $V$ and some point $x \in X$ such that $x \in V(\Gamma)$. Since $\left\{\diamond p_{i}: i \in \mathbb{N}\right\} \subseteq \Gamma$, for every $i \in \mathbb{N}$, there is an $x_{i} \in X$ with both $x R x_{i}$ and $x_{i} \in V\left(p_{i}\right)$. Also, if $i \neq j$ then $x_{i} \neq x_{j}$, since $x \in V\left(\square\left(p_{i} \supset \neg p_{j}\right)\right)$. So $X$ is infinite.

The proof of Lemma 3.1 is an unravelling construction that can easily be strengthened to prove 
LEMMA 3.3. Any countable rooted reflexive transitive Kripke frame is the image of $2^{<\omega}$ under some interior map.

Proof. For each $n \geq 0$, let $\mathbb{N}^{n}$ be the set of sequences of natural numbers of length $n$. Let $\mathbb{N}^{<\omega}={ }_{\mathrm{df}} \bigcup_{n=0}^{\infty} \mathbb{N}^{n}$, that is, $\mathbb{N}^{<\omega}$ is the set of finite sequences of natural numbers. We use $s, s^{\prime}$, etc., to range over $\mathbb{N}^{<\omega}$. As with binary sequences, we use " $\frown$ " for concatenation and we say $s \leq s^{\prime}$ iff $s$ is an initial segment of $s^{\prime}$. We identify $\mathbb{N}^{<\omega}$ with the countably infinite rooted transitive reflexive Kripke frame $\left\langle\mathbb{N}^{<\omega}, \leq\right\rangle$.

Suppose that $\langle X, R\rangle$ is a countable Kripke frame with root $r$. For our desired result, it suffices to construct two surjective interior maps: $f: 2^{<\omega} \rightarrow \mathbb{N}^{<\omega}$ and $g: \mathbb{N}^{<\omega} \rightarrow X$. Then $g \circ f$ will be an interior map from $2^{<\omega}$ onto $X$.

To construct $f: 2^{<\omega} \rightarrow \mathbb{N}^{<\omega}$, we first define a function zero: $2^{<\omega} \rightarrow \mathbb{N}$, as follows: $z \operatorname{ero}(\Lambda)=0$; zero $(b 0)=z \operatorname{ero}(b)+1$; and zero $(b 1)=0$. Note that zero $(b)$ is simply the number of uninterrupted occurrences of 0 at the end of $b$ : for example, $z \operatorname{ero}(001101000)=3$, zero $(100001)=0$, and zero $(000100)=2$. We now define our function $f: 2^{<\omega} \rightarrow \mathbb{N}^{<\omega}: f(\Lambda)=\Lambda, f(b 0)=f(b)$, and $f(b 1)=f(b)^{\frown}$ zero $(b)$. It is straightforward to check that $f$ is a surjective interior map.

To construct $g: \mathbb{N}^{<\omega} \rightarrow X$, for each $x \in X$ let $s u c c_{x}$ be any function from $\mathbb{N}$ onto $R(x)$. And define $g(\Lambda)=r$, the root of $X$; and $g(s \neg n)=\operatorname{succ}_{g(s)}(n)$. It is straightforward to check that $g$ is a surjective interior map.

Together with the fact that any consistent set $\Gamma$ of formulas is satisfiable in some countable rooted reflexive transitive Kripke frame (Makinson, 1966), Lemma 3.1 entails

LEMMA 3.4. S4 is strongly complete for $2^{<\omega}$.

So to prove Theorem 2.2 in the case $X=\mathbb{Q}$, we only need

LEMMA 3.5. There is an interior map from $\mathbb{Q}$ onto $2^{<\omega}$.

Theorem 2.4 in van Benthem et al. (2006) is our Theorem 2.1 in the case $X=\mathbb{Q}$ : the proof in (van Benthem et al., 2006) includes a proof of our Lemma 3.5.

\$4. The failure of interior map strategies. One strategy for proving our main result might be to apply the strategy in Section $\S 3$ to more spaces: for each dense-in-itself metric space $X$, find an interior map from $X$ onto $2^{<\omega}$. Unfortunately, this will not work: there is no interior map from $\mathbb{R}$ or from $\mathcal{C}$, for example, onto $2^{<\omega}$ (Corollary 4.8, below). Our arguments in this section depend on the Baire Category Theorem - see Theorem 4.1 belowDugundji (1966) is a good source on Baire spaces and the Baire Category Theorem. In particular, a topological space $X$ is a Baire space if the intersection of each countable family of open dense sets in $X$ is dense in $X$.

Theorem 4.1. (Baire Category Theorem, Dugundji, 1966, p. 299, Theorem 4.1). Any complete metric space, for example, $\mathbb{R}$ or $\mathcal{C}$ is a Baire space.

Lemma 4.2. (Engelking, 1989, p. 270, Theorem 4.3.11). Any closed subset of a complete metric space is a complete metric space.

COROLlary 4.3. Any closed subset of a complete metric space is a Baire space.

Lemma 4.4. (Dugundji, 1966, p. 256, Exercise 2). Any open subset of a Baire space is a Baire space. 
COROLlary 4.5. Suppose that $O$ is an open subset and $C$ is a closed subset of a complete metric space $X$. Then $O \cap C$ is a Baire space.

In what follows, we identify $\mathbb{N}$ with the Kripke frame $\langle\mathbb{N}, \leq\rangle$. For each $n \in \mathbb{N}$, let $[n]={ }_{\mathrm{df}}\{m \in \mathbb{N}: n \leq m\}$ : the nonempty open subsets of $\mathbb{N}$ are those of the form $[n]$ for $n \in \mathbb{N}$.

LEMMA 4.6. There is no interior map from any Baire space onto $\mathbb{N}$. Thus, for example, there is no interior map from $\mathbb{R}$ or from $\mathcal{C}$ onto $\mathbb{N}$.

Proof. Suppose that $f$ is an interior map from a Baire space $X$ onto $\mathbb{N}$. For each $n \in \mathbb{N}$, let $O_{n}=f^{-1}[[n]]$. Note that each $O_{n}$ is open and dense in $X$, since each $[n]$ is open and dense in $\mathbb{N}$ and $f$ is an interior map. Thus, $\bigcap_{n=0}^{\infty} O_{n}$ is dense in $O$, since $X$ is a Baire space. But $\bigcap_{n=0}^{\infty} O_{n}$ must be empty, since it is the preimage of $\bigcap_{n=0}^{\infty}[n]$, which is empty.

LEMMA 4.7. There is an interior map from $2^{<\omega}$ onto $\mathbb{N}$.

Proof. For each sequence $b \in 2^{<\omega}$, let $\ell(b)=|b|$, the length of $b$. Note that $\ell$ is an interior map from $2^{<\omega}$ onto $\mathbb{N}$.

COROLLARY 4.8. There is no interior map from any Baire space onto $2^{<\omega}$. Thus, there is no interior map from $\mathbb{R}$ or from $\mathcal{C}$ onto $2^{<\omega}$.

As noted above, Corollary 4.8 blocks any attempt to prove Theorem 2.2 by finding, for each dense-in-itself metric space $X$, a surjective interior map from $X$ onto $2^{<\omega}$. A subtler way to use interior maps might be as follows: for each dense-in-itself metric space $X$, show that each consistent set of sentences is satisfiable in some Kripke frame that is the image of an interior map from $X$. Example 4.9 and Lemma 4.10 block this strategy.

EXAMPLE 4.9. Suppose that $p_{0}, p_{1}, p_{2}, \ldots$ are distinct propositional variables. Let $\Gamma=$

$$
\begin{aligned}
& \left\{p_{0}\right\} \\
& \cup\left\{\square\left(p_{i} \supset \diamond p_{j}\right): i<j\right\} \\
& \cup\left\{\square\left(p_{i} \supset \neg \nabla p_{j}\right): i>j\right\} \\
& \cup\left\{\square\left(\bigwedge_{i=0}^{n} \neg p_{i} \supset \neg \diamond p_{n}\right): n \in \mathbb{N}\right\} .
\end{aligned}
$$

To see that $\Gamma$ is satisfiable, and therefore consistent, let $M=\langle\mathbb{N}, V\rangle$ where $\mathbb{N}$ is identified with the Kripke frame $\langle\mathbb{N}, \leq\rangle$ and where $V\left(p_{n}\right)=\{n\}$. Note that $V(\Gamma)=\{0\} .{ }^{10}$

LeMma 4.10. Suppose that $X$ is a complete metric space and $\Gamma$, as defined in Example 4.9, is satisfiable in the Kripke frame $\langle Y, R\rangle$. Then there is no interior map from $X$ onto $Y$.

Proof. Suppose that $X, \Gamma$ and $\langle Y, R\rangle$ are as described, and that $f: X \rightarrow Y$ is a surjective interior map. We will define a subspace $Y_{\geq 0}$ of $Y$, and a surjective interior map $g: Y_{\geq 0} \rightarrow \mathbb{N}$. We will then let $X^{*}={ }_{\mathrm{df}} f^{-1}\left[Y_{\geq 0}\right]$, and show that $X^{*}$ is a Baire space. Finally, we let $f^{*}$ be $f$ restricted to $X^{*}$ and show that $g \circ f^{*}$ is an interior map from $X^{*}$ onto $\mathbb{N}$, contradicting Lemma 4.6.

10 An anonymous referee alerted me to the similarity of the formulas in $\Gamma$ to the frame formulas of Fine (1974). 
Since $\Gamma$ is satisfiable in $\langle Y, R\rangle$, there is a valuation $V: P V \rightarrow \mathcal{P}(Y)$ and a $y_{0} \in Y$ such that $y_{0} \in V(\Gamma)$. We begin by defining certain subsets of $Y$ :

$$
\begin{aligned}
Y_{n} & ={ }_{\mathrm{df}} R\left(y_{0}\right) \cap V\left(p_{n}\right) \\
Y_{\geq n} & ={ }_{\mathrm{df}} \bigcup_{m \geq n} Y_{m} \quad=R\left(y_{0}\right) \cap \bigcup_{m \geq n} V\left(p_{m}\right) \\
Y_{\omega} & ={ }_{\mathrm{df}} R\left(y_{0}\right) \cap \bigcap_{n} V\left(\neg p_{n}\right) .
\end{aligned}
$$

Let $R_{\geq 0}$ be $R$ restricted to $Y_{\geq 0}$ : we will identify $Y_{\geq 0}$ with the Kripke frame $\left\langle Y_{\geq 0}, R_{\geq 0}\right\rangle$ and, through the identification of Kripke frames with Alexandroff spaces (as in Section §2), with the topological space $\left\langle Y_{\geq 0}, \tau_{R_{\geq 0}}\right\rangle$.

Claim 4.11. The $Y_{n}$ 's form a partition of $Y_{\geq 0}$. Proof. The fact that each $Y_{n}$ is nonempty follows from the fact that $y_{0} \in V\left(p_{0}\right)$ and $y_{0} \in V\left(\square\left(p_{0} \supset \diamond p_{n}\right)\right)$. To see that the $Y_{n}$ 's are pairwise disjoint, consider $Y_{i} \cap Y_{j}$, where $i>j$. The claim that $Y_{i} \cap Y_{j}=\emptyset$ follows from the fact that $y_{0} \in V\left(\square\left(p_{i} \supset \neg \vee p_{j}\right)\right)$.

Define $g: Y_{\geq 0} \rightarrow \mathbb{N}$ as follows: $g(y)=n$ iff $y \in Y_{n}$. Claim 4.11 implies

CLAIM 4.12. The function $g$ is well-defined for each $y \in Y_{\geq 0}$ and is surjective.

Claim 4.13. The function $g$ is continuous. Proof. Since $Y_{\geq 0}=\left\langle Y_{\geq 0}, R_{\geq 0}\right\rangle$ and $\mathbb{N}=$ $\langle\mathbb{N}, \leq\rangle$ are Kripke frames, it suffices to show that $g$ is monotonic: for $y, y^{\prime} \in Y_{\geq 0}$, if $y R_{\geq 0} y^{\prime}$, then $g(y) \leq g\left(y^{\prime}\right)$ (see Footnote 8). So suppose that $y, y^{\prime} \in Y_{\geq 0}$ and $y R_{\geq 0} y^{\prime}$, in wich case $y R y^{\prime}$. Let $m=g(y)$ and $k=g\left(y^{\prime}\right)$ so that $y \in Y_{m} \subseteq V\left(p_{m}\right)$ and $y^{\prime} \in Y_{k} \subseteq$ $V\left(p_{k}\right)$. And assume for a reductio that $k<m$. Note that $y \in V\left(\bigwedge_{i=0}^{k} \neg p_{i}\right)$, since $y_{0} R y$ and $y \in Y_{m} \subseteq V\left(p_{m}\right)$ and $y_{0} \in V\left(\square\left(p_{m} \supset \neg \diamond p_{i}\right)\right)$, for every $i<m$. So $y \in V\left(\neg \diamond p_{k}\right)$, since $y_{0} \in V\left(\square\left(\bigwedge_{i=0}^{k} \neg p_{i} \supset \neg \diamond p_{k}\right)\right)$. So $y^{\prime} \in V\left(\neg p_{k}\right)$, a contradiction.

Claim 4.14. The function $g$ is open. Proof. Since each of $Y_{\geq 0}=\left\langle Y_{\geq 0}, R_{\geq 0}\right\rangle$ and $\mathbb{N}=\langle\mathbb{N}, \leq\rangle$ are Kripke frames, it suffices to show the following, for any $y \in Y_{\geq 0}$ and any $n \in \mathbb{N}$ : if $g(y) \leq n$, then $g\left(y^{\prime}\right)=n$ for some $y^{\prime} \in Y_{\geq 0}$ with $y R_{\geq 0} y^{\prime}$ (see Footnote 9). So suppose that $g(y) \leq n$. Let $m=g(y)$, so that $y \in Y_{m} \subseteq V\left(p_{m}\right)$. Note that $y \in V\left(\diamond p_{n}\right)$ since $y_{0} \in V\left(\square\left(p_{m} \supset \diamond p_{n}\right)\right)$ and $y_{0} R y$ and $y \in V\left(p_{m}\right)$. So there is some $y^{\prime}$ with $y R y^{\prime}$ and $y^{\prime} \in V\left(p_{n}\right)$. Since $y_{0} R y$ and $y R y^{\prime}$, we have $y_{0} R y^{\prime}$ : thus $y^{\prime} \in R\left(y_{0}\right)$. So $y^{\prime} \in Y_{n}=R\left(y_{0}\right) \cap V\left(p_{n}\right)$. So $y^{\prime} \in Y_{\geq 0}$. So $y R_{\geq 0} y^{\prime}$, since $y R y^{\prime}$. Also $g\left(y^{\prime}\right)=n$, since $y^{\prime} \in Y_{n}$.

Claim 4.15. The function $g$ is an interior map from $Y_{\geq 0}$ onto $\mathbb{N}$. Proof. From Claims 4.12, 4.13, and 4.14.

Let $X^{*}=f^{-1}\left[Y_{\geq 0}\right]$.

Claim 4.16. $X^{*}$ is a Baire space. Proof. Given that $X$ is a complete metric space, it suffices by Lemma 4.5 to show that $X^{*}$ is the difference between two open sets in $X$. Given that $f: X \rightarrow Y$ is continuous, it suffices to show that $Y_{\geq 0}$ is the difference between two open sets in $Y$. Note that $Y_{\geq 0}=R\left(y_{0}\right)-Y_{\omega}$. Since $R\left(y_{0}\right)$ is open in $Y$, it suffices to show that $Y_{\omega}$ is open in $Y$, that is, that $Y_{\omega}$ is closed under $R$. So suppose that $y \in Y_{\omega}$ and $y R y^{\prime}$. Since $y \in Y_{\omega}$, we have $y_{0} R y$. So $y_{0} R y^{\prime}$. So $y^{\prime} \in R\left(y_{0}\right)$. It remains to show that $y^{\prime} \in V\left(\neg p_{n}\right)$ for each $n \in \mathbb{N}$. Fix $n \in \mathbb{N}$. Since $y \in Y_{\omega}$, we have $y \in V\left(\bigwedge_{i=0}^{n} \neg p_{i}\right)$. Also, $y_{0} \in V\left(\square\left(\bigwedge_{i=0}^{n} \neg p_{i} \supset \neg \vee p_{n}\right)\right)$. So $y \in V\left(\neg \diamond p_{n}\right)$. So, since $y R y^{\prime}, y^{\prime} \in V\left(\neg p_{n}\right)$, as desired.

Claim 4.17. Let $f^{*}$ be $f$ restricted to $X^{*}$. 
Claim 4.18. The function $g \circ f^{*}$ is an interior map from $X^{*}$ onto $\mathbb{N}$. Proof. Given Claim 4.15, it suffices to show that $f^{*}: X^{*} \rightarrow Y_{\geq 0}$ is a surjective interior map. To see that $f^{*}$ is surjective, note that $f^{*}\left[X^{*}\right]=f\left[X^{*}\right]=f\left[f^{-1}\left[Y_{\geq 0}\right]\right]=Y_{\geq 0}$. To see that $f^{*}$ is continuous, note that the restriction of a continuous function is continuous (see Dugundji, 1966, Theorem 8.2 (2) and (3)). To see that $f^{*}$ is open, let $O \subseteq X^{*}$ be an open set in $X^{*}$. Then $O=O^{\prime} \cap X^{*}$, for some set $O^{\prime} \subseteq X$ which is open in $X$. Since $f$ is open, $f\left[O^{\prime}\right]$ is open in $Y$. So $f\left[O^{\prime}\right] \cap Y_{\geq 0}$ is open in $Y_{\geq 0}$. So it suffices to show that $f^{*}[O]=f\left[O^{\prime}\right] \cap Y_{\geq 0}$. Note that $f^{*}[O]=f[O]$, since $O \subseteq X^{*}$ and $f^{*}=\left.f\right|_{X^{*}}$. Thus, $f^{*}[O]=f[O]=f\left[O^{\prime} \cap X^{*}\right] \subseteq f\left[O^{\prime}\right] \cap f\left[X^{*}\right]=f\left[O^{\prime}\right] \cap Y_{\geq 0}$. So to show that $f^{*}[O]=f\left[O^{\prime}\right] \cap Y_{\geq 0}$, it suffices to show that $f\left[O^{\prime}\right] \cap f\left[X^{*}\right] \subseteq f\left[O^{\prime} \cap X^{*}\right]$. So suppose that $y \in f\left[O^{\prime}\right] \cap f\left[X^{*}\right]$. Then $y=f(x)$ for some $x \in O^{\prime}$. Note that $x \in f^{-1}\left[Y_{\geq 0}\right]$, so that $x \in X^{*}$. So $x \in O^{\prime} \cap X^{*}$. So $y \in f\left[O^{\prime} \cap X^{*}\right]$, as desired.

Note that Claims 4.16 and 4.17 together contradict Lemma 4.6.

§5. Algebraic semantics. The remainder of the paper is devoted to proving our main result, Theorem 2.2. S4 is strongly complete for every dense-in-itself metric space. Here we introduce an algebraic generalization of the topological semantics. Except where indicated, the algebraic semantics here is a notational variant of the algebraic semantics in Rasiowa \& Sikorski (1963, Chapter XI, Section 8).

A Boolean algebra is a bounded complemented distributive lattice, that is, a partial order $\langle\mathbf{A}, \leq\rangle$ where

1. any $a, b \in \mathbf{A}$ have a least upper bound $a \sqcup b$ and a greatest lower bound $a \sqcap b(\langle\mathbf{A}, \leq\rangle$ is a lattice);

2. there is a least element 0 and a greatest element $1(\langle\mathbf{A}, \leq\rangle$ is bounded);

3. $\sqcup$ and $\sqcap$ distribute over each other, that is, for any $a, b, c \in \mathbf{A}$, we have $a \sqcup(b \sqcap c)=$ $(a \sqcup b) \sqcap(a \sqcup c)$ and $a \sqcap(b \sqcup c)=(a \sqcap b) \sqcup(a \sqcap c)(\langle\mathbf{A}, \leq\rangle$ is distributive $)$; and

4. for each $a \in \mathbf{A}$, there is a $-a \in \mathbf{A}$ such that both $a \sqcup-a=1$ and $a \sqcap-a=0$ ( $\langle\mathbf{A}, \leq\rangle$ is complemented). Given (3), $-a$ is unique.

A Boolean algebra is degenerate iff $0=1$.

An interior algebra is an ordered triple $\mathcal{I}=\langle\mathbf{A}, \leq, \mathbf{I}\rangle$ where $\langle\mathbf{A}, \leq\rangle$ is a Boolean algebra and $\mathbf{I}$ is a unary operator on $\mathbf{A}$ such that, for every $a, b \in \mathbf{A}$,

$$
\begin{aligned}
\mathbf{I} a & \leq a \\
\mathbf{I}(a & =\mathbf{I} a \\
\mathbf{I}(a \sqcap b) & =\mathbf{I} a \sqcap \mathbf{I} b \\
\mathbf{I} 1 & =1
\end{aligned}
$$

$\mathcal{I}=\langle\mathbf{A}, \leq, \mathbf{I}\rangle$ is degenerate iff $\langle\mathbf{A}, \leq\rangle$ is. If $X$ is a topological space, then the interior algebra of subsets of $X$ is the nondegenerate interior algebra $\mathcal{I}(X)=\left\langle\mathcal{P}(X), \subseteq, \operatorname{Int}_{X}\right\rangle$. If $\mathcal{I}=\langle\mathbf{A}, \leq \mathbf{I}\rangle$ and $\mathcal{I}^{\prime}=\left\langle\mathbf{A}^{\prime}, \leq^{\prime}, \mathbf{I}^{\prime}\right\rangle$ are interior algebras, then $\mathcal{I}^{\prime}$ is a subalgebra of $\mathcal{I}$ iff $\mathbf{A}^{\prime} \subseteq \mathbf{A}$ and the operations $\sqcup^{\prime}, \Pi^{\prime},-^{\prime}$ and $\mathbf{I}^{\prime}$ are $\sqcup, \sqcap,-$ and $\mathbf{I}$ restricted to $\mathbf{A}^{\prime}$. Note that, in this case, $0^{\prime}=0$ and $1^{\prime}=1$ and $\leq^{\prime}$ is $\leq$ restricted to $\mathbf{A}^{\prime} .{ }^{11}$

11 Interior algebras go back to their duals, the closure algebras of McKinsey \& Tarski (1944): Boolean algebras enriched with a closure operator rather than an interior operator. Interior algebras are called topological Boolean algebras in Rasiowa \& Sikorski (1963). The name "interior algebra" first appears in Blok (1976). 
Suppose that $\mathcal{I}=\langle\mathbf{A}, \leq, \mathbf{I}\rangle$ and $\mathcal{I}^{\prime}=\left\langle\mathbf{A}^{\prime}, \leq^{\prime}, \mathbf{I}^{\prime}\right\rangle$ are interior algebras and $h: \mathbf{A} \rightarrow \mathbf{A}^{\prime}$. Then $h$ is a Boolean homomorphism from $\mathcal{I}$ into $\mathcal{I}^{\prime}$ iff, for every $a, b \in \mathbf{A}$, we have $h(a \sqcup b)=h a \sqcup^{\prime} h b ; h(a \sqcap b)=h a \Gamma^{\prime} h b$; and $h(-a)=-^{\prime} h a$. Note that, if $h$ is a Boolean homomorphism, we also have $h(0)=0^{\prime}$ and $h(1)=1^{\prime}$. If $h$ is a Boolean homomorphism from $\mathcal{I}$ into $\mathcal{I}^{\prime}$, then $h$ is continuous iff for every $a \in \mathbf{A}, h(\mathbf{I} a) \leq^{\prime} \mathbf{I}^{\prime}(h a)$ and is a homomorphism iff for every $a \in \mathbf{A}, h(\mathbf{I} a)=\mathbf{I}^{\prime}(h a)$. A one-one homomorphism from $\mathcal{I}$ into $\mathcal{I}^{\prime}$ is an embedding. A classic result of McKinsey \& Tarski (1944) is that every nondegenerate interior algebra can be embedded into $\mathcal{I}(X)$ for some topological space $X .^{12}$

If $X$ and $X^{\prime}$ are topological spaces and $f: X^{\prime} \rightarrow X$, then $f$ induces a function $h_{f}$ : $\mathcal{P}(X) \rightarrow \mathcal{P}\left(X^{\prime}\right)$ as follows: for each $S \subseteq X, h_{f}(S)=f^{-1}[S]$.

LEMMA 5.1. The function $h_{f}$ is a Boolean homomorphism from the interior algebra $\mathcal{I}(X)$ into the interior algebra $\mathcal{I}\left(X^{\prime}\right)$. If $f$ is surjective, then $h_{f}$ is one-one. If $f$ is continuous, then $h_{f}$ is continuous. If $f$ is an interior map, then $h_{f}$ is a homomorphism. And if $f$ is a surjective interior map, then $h_{f}$ is an embedding.

An algebraic model is an ordered pair $\mathbf{M}=\langle\mathcal{I}, V\rangle$, where $\mathcal{I}=\langle\mathbf{A}, \leq, \mathbf{I}\rangle$ is a nondegenerate interior algebra, and $V: P V \rightarrow \mathbf{A}$. $V$ is called an (algebraic) valuation. For any algebraic model $\mathbf{M}=\langle\mathcal{I}, V\rangle, V$ is extended to all formulas as follows: $V(\neg A)=-V(A)$; $V(A \& B)=V(A) \sqcap V(B), V(A \vee B)=V(A) \sqcup V(B)$, and $V(\square A)=\mathbf{I}(V(A))$.

Suppose that $A$ is a formula, that $\Gamma$ is a set of formulas, that $\mathcal{I}$ is a nondegenerate interior algebra, and that $\mathbf{M}=\langle\mathcal{I}, V\rangle$ is an algebraic model. We say that $A$ is valid in $\mathbf{M}$ iff $V(A)=1$ and valid in $\mathcal{I}$ iff $A$ is valid in $\langle\mathcal{I}, V\rangle$ for every valuation $V$. We say that $\mathrm{S} 4$ is complete for $\mathcal{I}$ iff $A \in \mathrm{S} 4$ for every formula $A$ valid in $\mathcal{I}$. We say that $\Gamma$ is satisfied in $\mathbf{M}=\langle\mathcal{I}, V\rangle$ iff the set $\{V(A): A \in \Gamma\}$ has a nonzero lower bound in $\mathcal{I}$; and that $\Gamma$ is satisfiable in $\mathcal{I}$ iff $\Gamma$ is satisfied in some algebraic model $\mathbf{M}=\langle\mathcal{I}, V\rangle$. Note $\mathrm{S} 4$ is complete for $\mathcal{I}$ iff every finite consistent set of formulas is satisfiable in $\mathcal{I}$. We say that $\mathrm{S} 4$ is strongly complete for $\mathcal{I}$ iff every consistent set of formulas is satisfiable in $\mathcal{I}{ }^{13}$

LEMMA 5.2. If $X$ is a topological space, then any set $\Gamma$ of formulas is satisfiable in $X$ iff $\Gamma$ is satifiable in the interior algebra $\mathcal{I}(X)$, and $\mathrm{S} 4$ is strongly complete for $X$ iff $\mathrm{S} 4$ is strongly complete for $\mathcal{I}(X)$.

The following lemma and corollary generalize Lemma 2.3 and Corollary 2.4:

Lemma 5.3. Suppose $\mathbf{M}=\langle\mathcal{I}, V\rangle$ and $\mathbf{M}^{\prime}=\left\langle\mathcal{I}^{\prime}, V^{\prime}\right\rangle$ are algebraic models and that $h$ is an embedding from $\mathcal{I}$ into $\mathcal{I}^{\prime}$. Then for every formula $A, h(V(A))=V^{\prime}(A)$.

COROLlary 5.4. (Transfer of the strong completeness of S4, forwards via an embedding). Suppose $\mathcal{I}$ and $\mathcal{I}^{\prime}$ are nondegenerate interior algebras, and that there is an embedding from $\mathcal{I}$ into $\mathcal{I}^{\prime}$. Then if $\Gamma$ is satisfiable in $\mathcal{I}$, then $\Gamma$ is satisfiable in $\mathcal{I}^{\prime}$. Thus, if $\mathrm{S} 4$ is strongly complete for $\mathcal{I}$, then $\mathrm{S} 4$ is strongly complete for $\mathcal{I}^{\prime}$.

12 Theorem 2.4 in McKinsey \& Tarski (1944) makes this point in terms of closure algebras. This extends a classic result of Stone (1936), namely that every Boolean algebra can be embedded into $\langle\mathcal{P}(X), \subseteq\rangle$ for some set $X$.

13 We have not seen satisfiability and strong completeness for the algebraic semantics defined in the literature, certainly not in Rasiowa \& Sikorski (1963). 
Given Lemma 5.2, for our main result, Theorem 2.2, it suffices to show

THEOREM 5.5. If $X$ is a dense-in-itself metric space, then $\mathrm{S} 4$ is strongly complete for $\mathcal{I}(X)$.

Given Lemmas 3.4 and 5.2, we know

LEMMA 5.6. S4 is strongly complete for $\mathcal{I}\left(2^{<\omega}\right)$.

So, given Corollary 5.4, for Theorem 5.5, it suffices to show

LEMMA 5.7. If $X$ is a dense-in-itself metric space, then there is an embedding from $\mathcal{I}\left(2^{<\omega}\right)$ into $\mathcal{I}(X)$.

We prove Lemma 5.7 in Section $§ 9$.

§6. Strong completeness for the infinite binary tree with limits. In Section $\S 3$, we identified $2^{<\omega}$ with the infinite binary tree, that is, the countably infinite rooted transitive reflexive Kripke frame $\left\langle 2^{<\omega}, \leq\right\rangle$. Here we impose a topology on $2^{\leq \omega}$, the infinite binary tree with limits. For any $b \in 2^{<\omega}$, define $\llbracket b \rrbracket={ }_{\mathrm{df}}\left\{\boldsymbol{b}^{\prime} \in 2^{\leq \omega}: b \leq \boldsymbol{b}^{\prime}\right\}$. (Note that $\llbracket b \rrbracket \supsetneq[b])$. And take as a basis for our topology on $2^{\leq \omega}$ the following family of sets: $\left\{\llbracket b \rrbracket: b \in 2^{<\omega}\right\}$. It is easy to check that this is the Scott topology on $2^{\leq \omega}$, as defined, for example, in Gierz et al. (2003, p. 104). ${ }^{14}$

LEMMA 6.1. S4 is strongly complete for the topological space $2^{\leq \omega}$.

Before we prove Lemma 6.1, we note that Lando \& Sarenac (2011) prove that S4 is complete for $2^{\leq \omega}$-in particular, by showing that every finite Kripke frame is the image of an interior map from $2 \leq \omega$. Example 3.2, above, shows that for strong completeness, we need to work with infinite Kripke frames. We might hope to prove Lemma 6.1 by adapting the strategy in Lando \& Sarenac (2011) and showing that every Kripke frame whatsoever is the image of an interior map from $2^{\leq \omega}$. But this fails:

LEMMA 6.2. There is no interior map from $2^{\leq \omega}$ to $\mathbb{N}=\langle\mathbb{N}, \leq\rangle$.

Proof. This follows from Theorem 4.1 and Lemma 4.6 and Lemma 8.1, below.

Still, we might try another interior map strategy, similar to that suggested in Section $\S 4$ : find, for each consistent set $\Gamma$ of sentences, a Kripke frame in which $\Gamma$ is satisfiable and that is the image of an interior map from $2^{\leq \omega}$. But this will not work either:

LEMma 6.3. If $\Gamma$, as defined in Example 4.9, is satisfiable in the Kripke frame $\langle Y, R\rangle$, then there is no interior map from $2^{\leq \omega}$ onto $Y$.

Proof. This follows from Theorem 4.1, Lemma 4.10, and Lemma 8.1, below.

Happily, we can use the algebraic semantics of Section $\S 5$ to prove Lemma 6.1: it will suffice, given Lemma 5.2 and Corollary 5.4, to define an embedding from the interior algebra $\mathcal{I}\left(2^{<\omega}\right)$ into the interior algebra $\mathcal{I}\left(2^{\leq \omega}\right)$. In fact, we will define a family of such

14 Suppose that $X$ is a nonempty set partially ordered by $\leq$. A set $S \subseteq X$ is directed iff $S$ is nonempty and any finite subset of $S$ has an upper bound in $S . X$ is a directed complete partial order (DCPO) if every directed set has a least upper bound. Note: $2^{\leq \omega}$ is a DCPO, but $2^{<\omega}$ is not. A subset $O$ of a DCPO $X$ is Scott open iff it is both upper closed, that is, $(\forall x, y \in X)(x \in O \& x \leq y \Rightarrow y \in O)$; and for every directed set $S$, if $\sup (S) \in O$, then $S \cap O \neq \emptyset$. The Scott open sets form a topology, the Scott topology. 
embeddings. Suppose that $\mathcal{U}$ is a free ultrafilter on $\mathbb{N} .{ }^{15}$ Say that a set $S \subseteq 2^{<\omega} \mathcal{U}$-converges to $\mathbf{b} \in 2^{\omega}$ iff $\left\{n \in \mathbb{N}:\left.\mathbf{b}\right|_{n} \in S\right\} \in \mathcal{U}$. We write $S \longrightarrow \mathcal{U} \mathbf{b}$. Define $h_{\mathcal{U}}: \mathcal{P}\left(2^{<\omega}\right) \rightarrow \mathcal{P}\left(2^{\leq \omega}\right)$ as follows: for $S \subseteq 2^{<\omega}$, let $h_{\mathcal{U}}(S)=S \cup\left\{\mathbf{b} \in 2^{\omega}: S \longrightarrow \mathcal{U} \mathbf{b}\right\}$. We will freely use the fact that for $S \subseteq 2^{<\omega}, h_{\mathcal{U}}(S) \cap 2^{<\omega}=S$. Lemma 6.1 follows from Lemma 5.2, Corollary 5.4 , and

LEMMA 6.4. For each free ultrafilter $\mathcal{U}$ on $\mathbb{N}, h_{\mathcal{U}}$ is an embedding from $\mathcal{I}\left(2^{<\omega}\right)$ into $\mathcal{I}\left(2^{\leq \omega}\right)$.

Proof.

Claim 6.5. $h_{\mathcal{U}}\left(S \cap S^{\prime}\right)=h_{\mathcal{U}}(S) \cap h_{\mathcal{U}}\left(S^{\prime}\right)$. Proof. Note that for every $b \in 2^{<\omega}$, we have $b \in h_{\mathcal{U}}\left(S \cap S^{\prime}\right)$ iff $b \in\left(S \cap S^{\prime}\right)$ iff $b \in h_{\mathcal{U}}(S) \cap h_{\mathcal{U}}\left(S^{\prime}\right)$. So it suffices to show that for every $\mathbf{b} \in 2^{\omega}$, we have $\mathbf{b} \in h_{\mathcal{U}}\left(S \cap S^{\prime}\right)$ iff $\mathbf{b} \in h_{\mathcal{U}}(S) \cap h_{\mathcal{U}}\left(S^{\prime}\right)$. Choose $\mathbf{b} \in 2^{\omega}$. Note the following, where the "iff" marked with * is justified by the fact that $\mathcal{U}$ is a filter:

$\mathbf{b} \in h_{\mathcal{U}}\left(S \cap S^{\prime}\right)$

iff $\left(S \cap S^{\prime}\right) \longrightarrow \mathcal{U} \mathbf{b}$

iff $\left\{n \in \mathbb{N}:\left.\mathbf{b}\right|_{n} \in\left(S \cap S^{\prime}\right)\right\} \in \mathcal{U}$

iff $\left(\left\{n \in \mathbb{N}:\left.\mathbf{b}\right|_{n} \in S\right\} \cap\left\{n \in \mathbb{N}:\left.\mathbf{b}\right|_{n} \in S^{\prime}\right\}\right) \in \mathcal{U}$

iff* $\left\{n \in \mathbb{N}:\left.\mathbf{b}\right|_{n} \in S\right\} \in \mathcal{U}$ and $\left\{n \in \mathbb{N}:\left.\mathbf{b}\right|_{n} \in S^{\prime}\right\} \in \mathcal{U}$,

iff $S \longrightarrow \mathcal{U} \mathbf{b}$ and $S^{\prime} \longrightarrow \mathcal{U} \mathbf{b}$

iff $\mathbf{b} \in h_{\mathcal{U}}(S)$ and $\mathbf{b} \in h_{\mathcal{U}}\left(S^{\prime}\right)$

iff $\mathbf{b} \in h_{\mathcal{U}}(S) \cap h_{\mathcal{U}}\left(S^{\prime}\right)$.

Claim 6.6. $h_{\mathcal{U}}\left(2^{<\omega}-S\right)=2^{\leq \omega}-h_{\mathcal{U}}(S)$. Proof. Note that for every $b \in 2^{<\omega}$, we have $b \in h_{\mathcal{U}}\left(2^{<\omega}-S\right)$ iff $b \in\left(2^{<\omega}-S\right)$ iff $b \in 2^{\leq \omega}-h_{\mathcal{U}}(S)$. So it suffices to show that for every $\mathbf{b} \in 2^{\omega}$, we have $\mathbf{b} \in h_{\mathcal{U}}\left(2^{<\omega}-S\right)$ iff $\mathbf{b} \in 2^{\leq \omega}-h_{\mathcal{U}}(S)$. Choose $\mathbf{b} \in 2^{\omega}$. Note the following, where the iff marked with $*$ is justified by the fact that $\mathcal{U}$ is an ultrafilter:

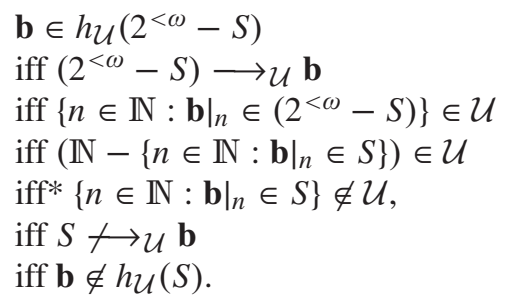

CLAim 6.7. $h_{\mathcal{U}}\left(S \cup S^{\prime}\right)=h_{\mathcal{U}}(S) \cup h_{\mathcal{U}}\left(S^{\prime}\right)$. This follows from Claims 6.5 and 6.6.

By Claims 6.5, 6.6, and 6.7, $h_{\mathcal{U}}$ is a Boolean homomorphism.

Claim 6.8. For each $b \in \operatorname{Int}_{2}<\omega(S)$ and $\mathbf{b} \in 2^{\omega}$, if $b \leq \mathbf{b}$, then $\mathbf{b} \in h_{\mathcal{U}}(S)$. Proof. Suppose that $b \in 2^{<\omega}, \mathbf{b} \in 2^{\omega}, b \in \operatorname{Int}_{2}<\omega(S)$ and $b \leq \mathbf{b}$. Since $b \leq \mathbf{b}, b=\left.\mathbf{b}\right|_{k}$ for

15 We say that a nonempty $\mathcal{F} \subseteq \mathcal{P}(\mathbb{N})$ is a filter on $\mathbb{N}$ iff, for any $S, S^{\prime} \subseteq \mathbb{N}$, we have (1) $\emptyset \notin \mathcal{F}$, (2) if $S, S^{\prime} \in \mathcal{F}$, then $S \cap S^{\prime} \in \mathcal{F}$, and (3) if $S \in \mathcal{F}$ and $S \subseteq S^{\prime}$, then $S^{\prime} \in \mathcal{F}$. We say that $\mathcal{U} \subseteq \mathcal{P}(\mathbb{N})$ is an ultrafilter on $\mathbb{N}$ iff $\mathcal{U}$ is a filter on $\mathbb{N}$ and, for any $S \subseteq \mathbb{N}$, either $S \in \mathcal{U}$ or $\mathbb{N}-S \in \mathcal{U}$. It is well-known that, if $\mathcal{U}$ is an ultrafilter, then $S \cup S^{\prime} \in \mathcal{U}$ iff either $S \in \mathcal{U}$ or $S^{\prime} \in \mathcal{U}$. We say that $\mathcal{U}$ is a principal ultrafilter on $\mathbb{N}$ iff there is some $S \subseteq \mathbb{N}$ such that $\mathcal{U}=\left\{S^{\prime} \subseteq \mathbb{N}: S \subseteq S^{\prime}\right\}$. Otherwise, $\mathcal{U}$ is free. It is well-known that free ultrafilters exist, though the proof of this requires some (weak) version of the Axiom of Choice. Note that every free ultrafilter on $\mathbb{N}$ contains every cofinite subset of $\mathbb{N}$. Moreover, every infinite subset of $\mathbb{N}$ is a member of some free ultrafilter on $\mathbb{N}$. 
some $k \in \mathbb{N}$. Thus, for each $n \geq k$, we have $\left.\mathbf{b}\right|_{n} \in S$, since $\left.\mathbf{b}\right|_{k}=b \in \operatorname{Int}_{2}<\omega(S)$. Thus $\left\{n \in \mathbb{N}:\left.\mathbf{b}\right|_{n} \in S\right\}$ is cofinite. So $\left\{n \in \mathbb{N}:\left.\mathbf{b}\right|_{n} \in S\right\} \in \mathcal{U}$, since $\mathcal{U}$ is a free ultrafilter. So $S \longrightarrow \mathcal{U}$ b. So $\mathbf{b} \in h_{\mathcal{U}}(S)$.

Claim 6.9. For each $b \in \operatorname{Int}_{2}<\omega(S)$, $\llbracket b \rrbracket \subseteq h_{\mathcal{U}}(S)$. Proof. Suppose that $b \in 2^{<\omega}$ and $b \in \operatorname{Int}_{2}<\omega(S)$. By Claim 6.8, $\left(2^{\omega} \cap \llbracket b \rrbracket\right) \subseteq h_{\mathcal{U}}(S)$. Also, $\left(2^{<\omega} \cap \llbracket b \rrbracket\right)=[b] \subseteq S \subseteq h_{\mathcal{U}}(S)$. Thus $\llbracket b \rrbracket \subseteq h_{\mathcal{U}}(S)$, as desired.

Claim 6.10. $\operatorname{Int}_{2}<\omega(S) \subseteq \operatorname{Int}_{2} \leq \omega\left(h_{\mathcal{U}}(S)\right)$. Proof. Suppose that $b \in I n t_{2}<\omega(S)$. Then, by Claim 6.9, $\llbracket b \rrbracket \subseteq h_{\mathcal{U}}(S)$, in which case $\llbracket b \rrbracket \subseteq \operatorname{Int}_{2} \leq \omega\left(h_{\mathcal{U}}(S)\right)$, since $\llbracket b \rrbracket$ is open in $2 \leq \omega$. Thus $b \in \llbracket b \rrbracket \subseteq \operatorname{Int}_{2} \leq \omega\left(h_{\mathcal{U}}(S)\right)$.

Claim 6.11. $h_{\mathcal{U}}\left(\operatorname{Int}_{2}<\omega(S)\right) \subseteq \operatorname{Int}_{2} \leq \omega\left(h_{\mathcal{U}}(S)\right)$. Proof. Note that $h_{\mathcal{U}}\left(\operatorname{Int}_{2}<\omega(S)\right) \cap$

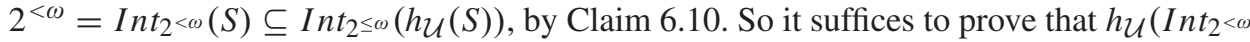

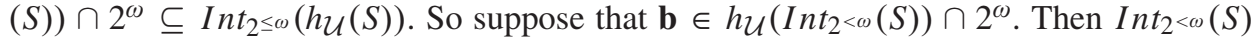
$\longrightarrow \mathcal{U}$ b. So $\left\{n \in \mathbb{N}:\left.\mathbf{b}\right|_{n} \in \operatorname{Int}_{2}<\omega(S)\right\} \in \mathcal{U}$. So $\left\{n \in \mathbb{N}:\left.\mathbf{b}\right|_{n} \in \operatorname{Int}_{2}<\omega(S)\right\}$ is nonempty. Choose $n \in \mathbb{N}$ with $\left.\mathbf{b}\right|_{n} \in I n t_{2}<\omega(S)$. Then by Claim 6.9, $\left.\llbracket \mathbf{b}\right|_{n} \rrbracket \subseteq h_{\mathcal{U}}(S)$, in which case

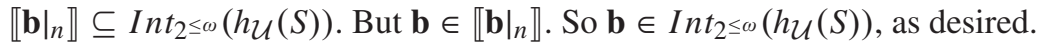

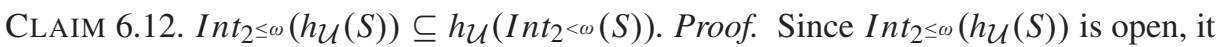
is the union of the basic sets that are subsets of it:

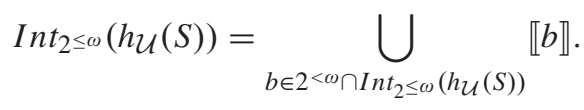

So it suffices to show that

$$
\llbracket b \rrbracket \subseteq h_{\mathcal{U}}\left(\operatorname{Int}_{2}<\omega(S)\right) \text {, for every } b \in 2^{<\omega} \cap \operatorname{Int}_{2} \leq \omega\left(h_{\mathcal{U}}(S)\right) .
$$

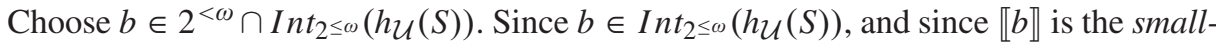
est open set in $2^{\leq \omega}$ containing $b$, we have $\llbracket b \rrbracket \subseteq h_{\mathcal{U}}(S)$. So $[b]=\llbracket b \rrbracket \cap 2^{<\omega} \subseteq h_{\mathcal{U}}(S) \cap$

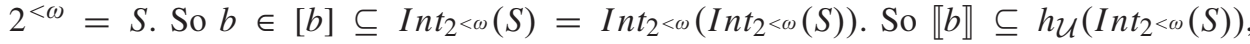
by Claim 6.9.

By Claims 6.5, 6.6, 6.7, 6.11 and 6.12, $h_{\mathcal{U}}$ is a homomorphism from $\mathcal{I}\left(2^{<\omega}\right)$ into $\mathcal{I}\left(2^{\leq \omega}\right)$.

Claim 6.13. $h_{\mathcal{U}}$ is one-one. Suppose that $h_{\mathcal{U}}(S)=h_{\mathcal{U}}\left(S^{\prime}\right)$. Then $S=h_{\mathcal{U}}(S) \cap 2^{<\omega}=$ $h_{\mathcal{U}}\left(S^{\prime}\right) \cap 2^{<\omega}=S^{\prime}$.

By Claims 6.5, 6.6, 6.7, 6.11, 6.12 and 6.13, $h_{\mathcal{U}}$ is an embedding from $\mathcal{I}\left(2^{<\omega}\right)$ into $\mathcal{I}\left(2^{\leq \omega}\right)$

6.1. The algebra of $\mathcal{U}$-convergent sets. Given a free ultrafilter $\mathcal{U}$ on $\mathbb{N}$, say that a set $S \subseteq 2^{\leq \omega}$ is $\mathcal{U}$-convergent iff $S \cap 2^{\omega}=\left\{\mathbf{b} \in 2^{\omega}: S \cap 2^{<\omega} \longrightarrow \mathcal{U} \mathbf{b}\right\}$. Let $\mathbf{A}_{\mathcal{U}}={ }_{\mathrm{df}}\left\{S \subseteq 2^{\leq \omega}:\right.$ $S$ is $\mathcal{U}$-convergent $\}$, let $\mathbf{I}_{\mathcal{U}}$ be the restriction of $\operatorname{Int}_{2} \leq \omega$ to $\mathbf{A}_{\mathcal{U}}$, and let $\mathcal{J}_{\mathcal{U}}=\mathrm{df}_{\mathrm{df}}\left\langle\mathbf{A}_{\mathcal{U}}, \subseteq, \mathbf{I}_{\mathcal{U}}\right\rangle$. Note that $\mathbf{A}_{\mathcal{U}}$ is the image of $\mathcal{P}\left(2^{<\omega}\right)$ under $h_{\mathcal{U}}$. Thus, $\mathcal{J}_{\mathcal{U}}$ is an interior algebra, indeed a proper subalgebra of $\mathcal{I}\left(2^{\leq \omega}\right)$. We call $\mathcal{J}$ the algebra of $\mathcal{U}$-convergent sets. Clearly, $h_{\mathcal{U}}$ is an embedding of $\mathcal{I}\left(2^{<\omega}\right)$ into (indeed onto) $\mathcal{J}_{\mathcal{U}}$. Thus, by Lemmas 3.4 and 5.2 and Corollary 5.4,

LEMMA 6.14. S4 is strongly complete for $\mathcal{J}_{\mathcal{U}}$.

\$7. For each dense-in-itself metric space $X$, a continuous function $f_{X}: X \rightarrow 2^{\leq \omega}$. The task of this section is construct, for each dense-in-itself metric space $X$, a continuous 
function $f \geqq_{X}: X \rightarrow 2^{\leq \omega}$. So fix a dense-in-itself metric space, $X$. We will begin by defining

1. nonempty open sets $O_{b} \subseteq X$, for each $b \in 2^{<\omega}$, with $b<b^{\prime} \Rightarrow O_{b^{\prime}} \subsetneq O_{b}$,

2. other nonempty sets $X_{b} \subsetneq O_{b}$, for each $b \in 2^{<\omega}$, and

3. possibly empty sets $X_{\mathbf{b}}=\bigcap_{n} O_{\left.\mathbf{b}\right|_{n}}$, for each $\mathbf{b} \in 2^{\omega}$.

This will deliver pairwise disjoint sets $X_{\boldsymbol{b}}$ for each $\boldsymbol{b} \in 2^{\leq \omega}$, and we will have $X=$ $\bigcup_{\boldsymbol{b} \in 2 \leq \omega} X_{\boldsymbol{b}}$. (The $X_{\boldsymbol{b}}$ will not necessarily form a partition of $X$, since there will be no guarantee that $X_{\boldsymbol{b}}$ is nonempty when $\boldsymbol{b} \in 2^{\omega}$.) We will then define the function $f_{X}: X \rightarrow$ $2^{\leq \omega}$ as follows: $f_{X}(x)$ is the unique $\boldsymbol{b} \in 2^{\leq \omega}$ with $x \in X_{\boldsymbol{b}}$. We will finally show that $f_{X}$ is continuous.

Our construction will rely on the decomposition lemma, Lemma 7.1, below. As an anonymous referee helpfully put it, this lemma allows us to produce Cantor-like subsets of any open set in a dense-in-itself metric space. In particular, the lemma allows us to decompose any open subset of a dense-in-itself metric space into three disjoint sets: a Cantor-like set, which we heuristically think of as "middle"; and two open sets, which we think of as "left" and "right". After we state the decomposition lemma, we provide Example 7.4 to show how this works when the initial open subset is the open unit interval, and middle is the Cantor set without endpoints.

Lemma 7.1. (The decomposition lemma, proved in Section $\S 10$., below). Suppose that $O \subseteq X$ is nonempty and open and that $\varepsilon>0$. Then there are pairwise disjoint nonempty sets $L(O, \varepsilon)$ ( $L$ for left), $R(O, \varepsilon)$ ( $R$ for right), and $M(O, \varepsilon)$ (M for middle) such that

1. $O=L(O, \varepsilon) \dot{\cup} R(O, \varepsilon) \dot{\cup} M(O, \varepsilon)$,

2. $L(O, \varepsilon)$ and $R(O, \varepsilon)$ are open,

3. $\partial(L(O, \varepsilon))$

$=\partial(R(O, \varepsilon))$

$=C l(M(O, \varepsilon))$

$=C l(O)-(L(O, \varepsilon) \cup R(O, \varepsilon))$, and

4. $(\forall x \in O)(d(x, M(O, \varepsilon)) \leq \varepsilon)$. (The E-clause. $)$

REMARK 7.2. Without the $\varepsilon$-clause, Lemma 7.1 is a special case of Theorem III, 7.1, in Rasiowa \& Sikorski (1963). We added the $\varepsilon$-clause for the proof of Lemma 9.1, below.

REMARK 7.3. Henceforth, we will assume that a choice has been made of $L, R$, and $M$, considered as functions from $\left(\tau_{X}-\{\emptyset\}\right) \times \mathbb{R}^{+}$to $\mathcal{P}(X)$, satisfying the clauses of Lemma 7.1. We could proceed, instead, by keeping track of the parameters $L, R$, and $M$ but that would require a profusion of subscripts, superscripts, or other similar devices.

EXAMPLE 7.4. Suppose that $O$ is the open unit interval $(0,1)$ and $\varepsilon=\frac{1}{6}$. Then we can let $M(O, \varepsilon)=M\left((0,1), \frac{1}{6}\right)$ be the Cantor set without endpoints, as in Figure 4. As for $\left((0,1), \frac{1}{6}\right)$ and $\left((0,1), \frac{1}{6}\right)$, recall that one way to construct the Cantor set is to delete the middle third of $(0,1)$, that is, delete $\left(\frac{1}{3}, \frac{2}{3}\right)$, and then to iterate this process, deleting the middle thirds of the remaining undeleted closed or semi-closed intervals. Label the deleted thirds with L (for 'left') and R (for right) as in Figure 5. We can then let $\left((0,1), \frac{1}{6}\right)$ be the union of the subintervals labelled with $\mathrm{L}$ and $\left((0,1), \frac{1}{6}\right)$ be the union of the subintervals labelled with R. Note that the clauses in Lemma 7.1 are all satisfied. 


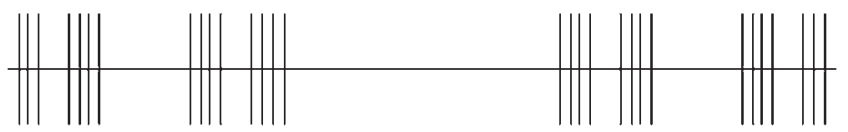

Fig. 4. One choice for $M\left((0,1), \frac{1}{6}\right)$ : the Cantor set without the endpoints 0 and 1 .

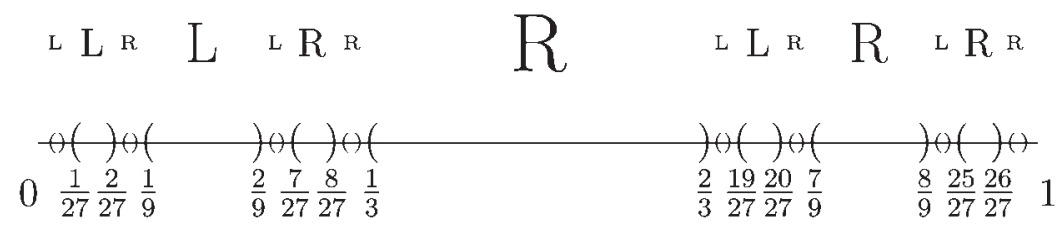

Fig. 5. Labelling deleted middle thirds with $\mathrm{L}$ and $\mathrm{R}$.

Given Lemma 7.1, we define the $O_{b}$, for $b \in 2^{<\omega}$, recursively as follows:

$$
\begin{aligned}
& O_{\Lambda}==_{\mathrm{df}} \quad X \\
& O_{b 0}==_{\mathrm{df}} L\left(O_{b}, \frac{1}{|b|+1}\right) \\
& O_{b 1}={ }_{\mathrm{df}} \quad R\left(O_{b}, \frac{1}{|b|+1}\right)
\end{aligned}
$$

For $b \in 2^{<\omega}$, we define

$$
X_{b}={ }_{\mathrm{df}} M\left(O_{b}, \frac{1}{|b|+1}\right) .
$$

Thus, $\left(\forall b \in 2^{<\omega}\right)\left(O_{b}=O_{b 0} \dot{\cup} O_{b 1} \dot{\cup} X_{b}\right){ }^{16}$

REMARK 7.5. Note our use of the $\varepsilon$-clause: the further along you go in the construction, the closer points in $O_{b}$ are guaranteed to be to $X_{b}$. For example, $\left(\forall x \in O_{1101011}\right)(d(x$, $\left.\left.X_{1101011}\right) \leq \frac{1}{7}\right)$. Of course, by making $|b|$ large, we can make this distance as small as we like: see (Case 2) in the proof of of Lemma 9.1.

Note the following facts about the $O_{b}$ and the $X_{b}$ :

LEMMA 7.6.

1. $X_{b}$ and $O_{b}$ are nonempty, for each $b \in 2^{<\omega}$.

2. If $b<b^{\prime}$, then $X_{b^{\prime}} \subsetneq O_{b^{\prime}} \subsetneq O_{b}$.

3. If $b<b^{\prime}$, then $X_{b} \cap X_{b^{\prime}}=X_{b} \cap O_{b^{\prime}}=\emptyset$.

4. If $b^{\prime} \not \leq b \not \leq b^{\prime}$, then $O_{b} \cap O_{b^{\prime}}=\emptyset$.

5. If $b \not b^{\prime}$, then $O_{b} \cap X_{b^{\prime}}=\emptyset$.

6. If $b \neq b^{\prime}$, then $X_{b} \cap X_{b^{\prime}}=\emptyset$.

LEMMA 7.7. $\left(\forall b, b^{\prime} \in 2^{<\omega}\right)\left(b \leq b^{\prime} \Rightarrow C l\left(X_{b}\right) \subseteq C l\left(X_{b^{\prime}}\right)\right)$.

Proof. Fix $b \in 2^{<\omega}$. We will show $\left(\forall b^{\prime} \in 2^{<\omega}\right)\left(b \leq b^{\prime} \Rightarrow C l\left(X_{b}\right) \subseteq C l\left(X_{b^{\prime}}\right)\right)$ by induction on the construction of $b^{\prime} \geq b$. For the base case, assume that $b^{\prime}=b$. Then, trivially, $C l\left(X_{b}\right) \subseteq C l\left(X_{b}^{\prime}\right)$.

16 The $O_{b}$ and the $X_{b}$ depend on much more than $b$ : they depend on the space $X$ as well as the choice of $L, R$, and $M$, from Lemma 7.1. Similar remarks apply to the $X_{\mathbf{b}}$, defined below, for $\mathbf{b} \in 2^{\omega}$. See Remark 7.3. 
For the inductive step, suppose that $C l\left(X_{b}\right) \subseteq C l\left(X_{b^{\prime}}\right)$. We must show both that $C l\left(X_{b}\right) \subseteq C l\left(X_{b^{\prime} 0}\right)$ and $C l\left(X_{b}\right) \subseteq C l\left(X_{b^{\prime} 1}\right)$. For these, it suffices to show that $C l\left(X_{b^{\prime}}\right) \subseteq$ $C l\left(X_{b^{\prime} 0}\right)$ and $C l\left(X_{b^{\prime}}\right) \subseteq C l\left(X_{b^{\prime} 1}\right)$. Note:

$$
\begin{array}{rlrl}
C l\left(X_{b^{\prime}}\right)= & \partial\left(O_{b^{\prime} 0}\right) & & \text { by Lemma 7.1, Clause 3, } \\
= & C l\left(O_{b^{\prime} 0}\right)-O_{b^{\prime} 0} & & \text { since } O_{b^{\prime} 0} \text { is open, } \\
= & C l\left(O_{b^{\prime} 0}\right) & & \\
& -\left(O_{b^{\prime} 00} \cup O_{b^{\prime} 01} \cup X_{b 0}\right) & \text { by Lemma 7.1, Clause 1, } \\
\subseteq & C l\left(O_{b^{\prime} 0}\right)-\left(O_{b^{\prime} 00} \cup O_{b^{\prime} 01}\right) & \\
= & C l\left(X_{b^{\prime} 0}\right) & \text { by Lemma 7.1, Clause 3. }
\end{array}
$$

Similarly, $C l\left(X_{b^{\prime}}\right) \subseteq C l\left(X_{b^{\prime} 1}\right)$.

For $\mathbf{b} \in 2^{\omega}$, define $X_{\mathbf{b}}={ }_{\mathrm{df}} \bigcap_{n \in \mathbb{N}} O_{\left.\mathbf{b}\right|_{n}}$. Notice that when the binary sequence $\mathbf{b}$ is infinite, there is no guarantee that $X_{\mathbf{b}}$ is nonempty. Now, we have defined $X_{\boldsymbol{b}}$ for each $\boldsymbol{b} \in 2^{\leq \omega}$. Note that the $X_{\boldsymbol{b}}$ are pairwise disjoint and that $X=\bigcup_{\boldsymbol{b} \in 2 \leq \omega} X_{\boldsymbol{b}}$. So every $x \in X$ is a member of exactly one $X_{\boldsymbol{b}}$. Define $f_{X}: X \rightarrow 2^{\leq \omega}$ as follows:

$$
f_{X}(x) \text { is the unique } \boldsymbol{b} \in 2^{\leq \omega} \text { with } x \in X_{\boldsymbol{b}} \cdot{ }^{17}
$$

LeMma 7.8. $\left(\forall b \in 2^{<\omega}\right)\left(\forall x \in O_{b}\right)\left(b \leq f_{X}(x)\right)$.

Proof. Choose $b \in 2^{<\omega}$ and $x \in O_{b}$. Suppose, for a reductio, that $b \leq f_{X}(x)$. We consider two cases. (Case 1) $f_{X}(x) \in 2^{<\omega}$. Then $O_{b} \cap X_{f_{X}(x)}=\emptyset$, since $b \not \leq f_{X}(x)$ and by Lemma 7.6, (5). But then, since $x \in X_{f_{X}(x)}$, we have $x \notin O_{b}$, a contradiction. (Case 2) $f_{X}(x) \in 2^{\omega}$. Let $n=|b|$. Then $b \neq\left. f_{X}(x)\right|_{n}$, since $b \not \leq f_{X}(x)$. So $b \not \leq\left. f_{X}(x)\right|_{n} \not \leq b$, since $b$ and $\left.f_{X}(x)\right|_{n}$ are of the same length. So $O_{b} \cap O_{\left.f_{X}(x)\right|_{n}}=\emptyset$. But $x \in X_{f_{X}(x)}=$ $\bigcap_{k \in \mathbb{N}} O_{\left.f_{X}(x)\right|_{k}} \subseteq O_{\left.f_{X}(x)\right|_{n}}$. So $x \notin O_{b}$, a contradiction.

LEMMA 7.9. The function $f_{X}: X \rightarrow 2^{\leq \omega}$ is continuous.

Proof. It suffices to show that the preimage, under $f_{X}$, of any basis set in the topology we have defined on $2^{\leq \omega}$ is open. So it suffices to show that $f_{X}{ }^{-1}[\llbracket b \rrbracket]=O_{b}$, for any $b \in 2^{<\omega}$.

Choose $b \in 2^{<\omega}$, and let $S=f_{X}^{-1}[\llbracket b \rrbracket]$. By Lemma 7.8, $O_{b} \subseteq S$. To prove that $S \subseteq O_{b}$, choose $x \in S$. Then $f_{X}(x) \in \llbracket b \rrbracket$, so that $b \leq f_{X}(x)$. Recall that $x \in X_{f_{X}(x)}$ : so, to show that $x \in O_{b}$, it suffices to show that $X_{f_{X}(x)} \subseteq O_{b}$. If $f_{X}(x) \in 2^{<\omega}$, then $X_{f_{X}(x)} \subseteq O_{b}$, by Lemma 7.6, Clause 2. Suppose, on the other hand, that $f_{X}(x) \in 2^{\omega}$. Since $b \leq f_{X}(x)$, we get $b=\left.f_{X}(x)\right|_{k}$ for some $k \in \mathbb{N}$. Thus, $X_{f_{X}(x)}=\bigcap_{n \in \mathbb{N}} O_{\left.f_{X}(x)\right|_{n}} \subseteq$ $O_{\left.f_{X}(x)\right|_{k}}=O_{b}$.

§8. Strong completeness for each complete dense-in-itself metric space. The main result of this section is

LEMMA 8.1. If $X$ is a complete dense-in-itself metric space, then $f_{X}: X \rightarrow 2^{\leq \omega}$ is a surjective interior map.

From Lemmas 8.1, 6.1, and Corollary 2.4, we get

17 As emphasized by an anonymous referee, this is somewhat imprecise notation, since the function $f_{X}$ depends not only on $X$ but also on the choice of $L, R$, and $M$, from Lemma 7.1. See Remark 7.3 and Footnote 16 above. 
THEOREM 8.2. S4 is strongly complete for any complete dense-in-itself metric space $X$.

Before we can prove Lemma 8.1, some preliminary notions. If $S$ is a nonempty subset of a metric space, then the diameter of $S$ is $\operatorname{diam}(S)={ }_{\mathrm{df}} \operatorname{supremum}\left\{d\left(x, x^{\prime}\right): x, x^{\prime} \in S\right\}$, if this set is bounded above. If $\left\{d\left(x, x^{\prime}\right): x, x^{\prime} \in S\right\}$ is not bounded above, then $\operatorname{diam}(S)=$ $\infty$. We will use the following lemma, a standard textbook exercise on metric spaces:

LEMMA 8.3. A metric space $X$ is complete iff every decreasing sequence of nonempty closed subsets of $X$ with diameters tending to 0 has a nonempty intersection.

Proof of Lemma 8.1. Suppose that $X$ is a complete dense-in-itself metric space. The function $f_{X}$ is continuous by Lemma 7.9. We need to prove that $f_{X}$ is an open surjection. Shortly, we will prove the following claim:

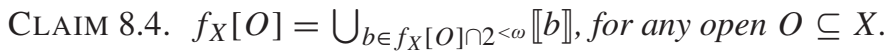

The openness of $f_{X}$ follows from Claim 8.4: the image of any open subset of $X$ is the union of open subsets of $2^{\leq \omega}$. The surjectivity of $f_{X}$ also follows from Claim 8.4: $f_{X}[X]=\bigcup_{b \in f_{X}[X] \cap 2^{<\omega}} \llbracket b \rrbracket \supseteq \llbracket \Lambda \rrbracket=2^{\leq \omega}$. Before we prove Claim 8.4, a preliminary claim:

CLAIM 8.5. For any open $O \subseteq X$, if $b \in f_{X}[O] \cap 2^{<\omega}$ and $b \leq b^{\prime} \in 2^{<\omega}$, then $\emptyset \neq O \cap X_{b^{\prime}} \subseteq O \cap O_{b}$.

Proof of Claim 8.5. Suppose that $O \subseteq X$ is open, $b \in f_{X}[O] \cap 2^{<\omega}, b^{\prime} \in 2^{<\omega}$ and $b \leq b^{\prime}$. The fact that $O \cap X_{b^{\prime}} \subseteq O \cap O_{b}$ follows from the fact that $X_{b^{\prime}} \subseteq O_{b}$ (Lemma 7.6, Clause (2)). To see that $O \cap X_{b^{\prime}} \neq \emptyset$, choose any $x \in O$ with $f_{X}(x)=b$, and note: $x \in X_{b} \subseteq C l\left(X_{b}\right) \subseteq C l\left(X_{b^{\prime}}\right)$, by Lemma 7.7. So $x \in O \cap C l\left(X_{b^{\prime}}\right)$. So $O \cap X_{b^{\prime}} \neq \emptyset$.

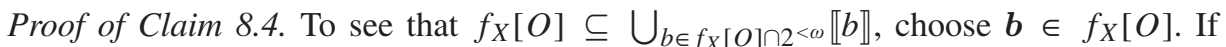

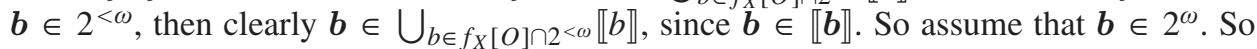
$\boldsymbol{b}=f_{X}(x)$ for some $x \in X_{\boldsymbol{b}} \cap O$. Choose $n \in \mathbb{N}$ such that $\mathbf{B}(x, 2 /(n+1)) \subseteq O$, and let

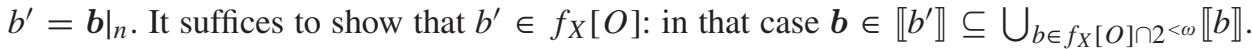
By the definition of $X_{\boldsymbol{b}}$, we have $x \in O_{b^{\prime}}$. And by the definition of $X_{b^{\prime}}$, we have $(\forall y \in$ $\left.O_{b^{\prime}}\right)\left(d\left(y, X_{b^{\prime}}\right) \leq 1 /\left(\left|b^{\prime}\right|+1\right)\right)$. So $d\left(x, X_{b^{\prime}}\right) \leq 1 /\left(\left|b^{\prime}\right|+1\right)<2 /(n+1)$. Choose $y \in X_{b^{\prime}}$ with $d(x, y)<2 /(n+1)$. Then $f_{X}(y)=b^{\prime}$ and $b^{\prime} \in \mathbf{B}(x, 2 /(n+1)) \subseteq O$. So $b^{\prime} \in f_{X}[O]$, as desired.

Now we use Claim 8.5 to show that $\bigcup_{b \in f_{X}[O] \cap 2<\omega} \llbracket b \rrbracket \subseteq f_{X}[O]$. So choose $b \in f_{X}[O] \cap$ $2^{<\omega}$ : we want to show that $\llbracket b \rrbracket \subseteq f_{X}[O]$. So choose $\boldsymbol{b}^{\prime} \in \llbracket b \rrbracket$ : we want to show that $\boldsymbol{b}^{\prime} \in f_{X}[O]$. On the one hand, if $\boldsymbol{b}^{\prime} \in 2^{<\omega}$, then $O \cap X_{\boldsymbol{b}^{\prime}}$ is nonempty, by Claim 8.5; so we can choose $x \in O \cap X_{\boldsymbol{b}^{\prime}}$ and note that $\boldsymbol{b}^{\prime}=f_{X}(x) \in f_{X}[O]$.

Suppose, on the other hand, that $\boldsymbol{b}^{\prime} \in 2^{\omega}$. Let $n=|b|$ so that $\left.\boldsymbol{b}^{\prime}\right|_{n}=b$. Choose any $x \in O$ such that $f_{X}(x)=b$. So $x \in O \cap O_{b}=O \cap O_{\left.b^{\prime}\right|_{n}}$, and $x \in X_{\left.\boldsymbol{b}^{\prime}\right|_{n}}$. We will now inductively define a decreasing sequence,

$$
C_{0} \supsetneq C_{1} \supsetneq \cdots \supsetneq C_{k} \supsetneq \cdots,
$$

of nonempty closed subsets of $X$ with diameters tending to 0 . Let $x_{0}=x$, and choose any positive $r_{0} \in \mathbb{R}^{+}$so that $C_{0}=_{\mathrm{df}} C l\left(\mathbf{B}\left(x_{0}, r_{0}\right)\right) \subsetneq O \cap O_{b}=O \cap O_{\left.\boldsymbol{b}^{\prime}\right|_{n}}$. Suppose that $r_{k} \in \mathbb{R}^{+}$and $x_{k} \in X_{\left.\boldsymbol{b}^{\prime}\right|_{n+k}}$ are such that $C_{k}=C l\left(\mathbf{B}\left(x_{k}, r_{k}\right)\right) \subsetneq O \cap O_{\left.\boldsymbol{b}^{\prime}\right|_{n+k}}$. Recall that $X_{\left.\boldsymbol{b}^{\prime}\right|_{n+k}} \subseteq C l\left(X_{\left.\boldsymbol{b}^{\prime}\right|_{n+k+1}}\right)$ (Lemma 7.7). So we can choose $x_{k+1} \in \mathbf{B}\left(x_{k}, r_{k}\right) \cap X_{\left.\boldsymbol{b}^{\prime}\right|_{n+k+1}}$. 
Note that $x_{k+1} \in \mathbf{B}\left(x_{k}, r_{k}\right) \cap O_{\left.\boldsymbol{b}^{\prime}\right|_{n+k+1}}$. So we can choose $r_{k+1} \leq r_{k} / 2$ such that $C_{k+1}=\mathrm{df}_{\mathrm{df}}$ $\mathrm{Cl}\left(\mathbf{B}\left(x_{k+1}, r_{k+1}\right)\right) \subsetneq \mathbf{B}\left(x_{k}, r_{k}\right) \cap O_{\left.\mathbf{b}^{\prime}\right|_{n+k+1}}$.

Notice that the each $C_{k}$ is closed and nonempty, and that the sequence of $C_{k}$ 's satisfies (*). Notice also that $\operatorname{diam}\left(C_{k}\right) \leq r_{k}$ and that $r_{k} \rightarrow 0$, so that $\operatorname{diam}\left(C_{k}\right) \rightarrow 0$. So, by Lemma 8.3, there is a $y \in \bigcap_{k} C_{k}$. Also note that each $C_{k} \subseteq O$, so that $y \in O$. Finally, notice that each $C_{k} \subseteq O_{\left.\boldsymbol{b}^{\prime}\right|_{n+k}}$, so that $y \in \bigcap_{k} O_{\left.\boldsymbol{b}^{\prime}\right|_{n+k}}$ But $\bigcap_{k} O_{\left.\boldsymbol{b}^{\prime}\right|_{n+k}}=\bigcap_{m} O_{\left.\boldsymbol{b}^{\prime}\right|_{m}}$, since the $O_{\left.\boldsymbol{b}^{\prime}\right|_{m}}$ 's are decreasing: $O_{\left.\boldsymbol{b}^{\prime}\right|_{0}} \supseteq O_{\left.\boldsymbol{b}^{\prime}\right|_{1}} \supseteq O_{\left.\boldsymbol{b}^{\prime}\right|_{2}} \supseteq \cdots$. So $y \in \bigcap_{m} O_{\left.\boldsymbol{b}^{\prime}\right|_{m}}=X_{\boldsymbol{b}^{\prime}}$. So $f_{X}(y)=\boldsymbol{b}(y)=\boldsymbol{b}^{\prime}$. So $\boldsymbol{b}^{\prime} \in f_{X}[O]$, as desired.

\$. Strong completeness for each dense-in-itself metric space. As seen in Section $\S 8$, if $X$ is a complete dense-in-itself metric space, then $f_{X}: X \rightarrow 2^{\leq \omega}$ is a surjective interior map: thus, strong completeness is transferred from $2^{\leq \omega}$ to $X$, backwards via $f_{X}$. Unfortunately, there is no general guarantee that $f_{X}$ will be a surjective interior map: for example, if the cardinality of $X$ is less than $2^{\aleph_{0}}$, then $f_{X}$ cannot be a surjection and also cannot be an interior map. ${ }^{18}$

9.1. Algebraic semantics to the rescue. Fix any free ultrafilter $\mathcal{U}$ on $\mathbb{N}$. Recall, from Section $\S 6$, the embedding $h_{\mathcal{U}}$ from $\mathcal{I}\left(2^{<\omega}\right)$ into $\mathcal{I}\left(2^{\leq \omega}\right)$. As noted in Section 6.1., $h_{\mathcal{U}}$ is also an embedding from $\mathcal{I}\left(2^{<\omega}\right)$ into the algebra $\mathcal{J} U$ of $\mathcal{U}$-convergent sets, for which $\mathrm{S} 4$ is therefore strongly complete (see Lemma 6.14).

Recall also that the function $f_{X}: X \rightarrow 2^{\leq \omega}$ induces a function $h_{f_{X}}: \mathcal{P}\left(2^{\leq \omega}\right) \rightarrow \mathcal{P}(X)$ as follows: for $S \in \mathcal{P}\left(2^{\leq \omega}\right), h_{f_{X}}(S)=f_{X}{ }^{-1}[S]$. Since $f_{X}$ is continuous, the function $h_{f_{X}}$ is guaranteed to be a continuous Boolean homomorphism from $\mathcal{I}\left(2^{\leq \omega}\right)$ into $\mathcal{I}(X)$, by Lemma 5.1. The function $h_{f_{X}}$ is not, however, guaranteed to be an embedding from $\mathcal{I}\left(2^{<\omega}\right)$ into $\mathcal{I}(X)$. But instead of considering $h_{f_{X}}$, let $h^{*}$ be the restriction of $h_{f_{X}}$ to $\mathbf{A}_{\mathcal{U}}{ }^{19}$

LEMMA 9.1. $h^{*}$ is an embedding from $\mathcal{J}_{\mathcal{U}}$ into $\mathcal{I}(X)$.

Proof. Since $h_{f_{X}}$ is a continuous Boolean homomorphism, so is $h^{*}$. That $h^{*}$ is an embedding follows from Claims 9.2 and 9.3, below.

Claim 9.2. The function $h^{*}$ is one-one. Proof. Consider $S, S^{\prime} \in \mathbf{A}_{\mathcal{U}}$, and suppose that $h^{*}(S)=h^{*}\left(S^{\prime}\right)$. So $f_{X}{ }^{-1}[S]=f_{X}{ }^{-1}\left[S^{\prime}\right]$. To show that $S=S^{\prime}$ it suffices to show that $S \cap 2^{<\omega}=S^{\prime} \cap 2^{<\omega}$, since $S$ and $S^{\prime}$ are $\mathcal{U}$-convergent. To see that $S \cap 2^{<\omega} \subseteq S^{\prime} \cap 2^{<\omega}$, let $b \in S \cap 2^{<\omega}$. Since $b \in 2^{<\omega}$, the set $X_{b}$ is nonempty (Lemma 7.6, (1)). Choose any $x \in X_{b}$. Note that $x \in f_{X}^{-1}[S]$, since $f_{X}(x)=b \in S$. So $x \in f_{X}^{-1}\left[S^{\prime}\right]$. So $b=f_{X}(x) \in S^{\prime}$. So $b \in S^{\prime} \cap 2^{<\omega}$, as desired. Similarly, $S^{\prime} \cap 2^{<\omega} \subseteq S \cap 2^{<\omega}$.

CLAIM 9.3. For every $\mathcal{U}$-convergent set $S \subseteq 2^{\leq \omega}$, we have $\operatorname{Int}_{X}\left(h^{*}(S)\right) \subseteq h^{*}\left(\operatorname{Int}_{2} \leq \omega(S)\right)$. Proof. Suppose that $S \subseteq 2^{\leq \omega}$ is $\mathcal{U}$-convergent, and also that $x \in \operatorname{Int}_{X}\left(h^{*}(S)\right)$.

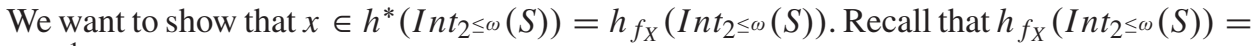
$f_{X}^{-1}\left[\operatorname{Int} t_{2} \leq \omega(S)\right]$. So we want to show that $f_{X}(x) \in \operatorname{Int}_{2} \leq \omega(S)$. First, since $x \in$

18 To see this second point, note that, by the definition of $f_{X}$, the empty sequence $\Lambda$ is in $f_{X}[X]$, that is, the range of $f_{X}$. If $f_{X}$ were an interior map, then the set $f_{X}[X]$ would have to be open in $2^{\leq \omega}$, but the only open set in $2^{\leq \omega}$ containing $\Lambda$ is $2^{\leq \omega}$ itself. So $f_{X}$ would have to be surjective. An anonymous referee conjectured that $f_{X}$ will always be an interior map from $X$ to $f_{X}[X]$, considered a subspace of $2^{\leq \omega}$. But nothing in the construction in Section $\S 7$ guarantees this.

19 The function $h^{*}: \mathbf{A}_{\mathcal{U}} \rightarrow \mathcal{P}(X)$ depends on a host of parameters: on the ultrafilter $\mathcal{U}$, on the topological space $X$, and even on $L, R$, and $M$ as in Footnotes 16 and 17. 
$\operatorname{Int}_{X}\left(h^{*}(S)\right)$, there is some positive $r \in \mathbb{R}$ such that $x \in \mathbf{B}(x, r) \subseteq h^{*}(S)=h_{f_{X}}(S)$. To show that $f_{X}(x) \in \operatorname{Int}_{2} \leq \omega(S)$, we consider two cases.

(Case 1) $f_{X}(x) \in 2^{<\omega}$. It suffices to show that $\llbracket f_{X}(x) \rrbracket \subseteq S$. That is, it suffices to show that $\left(\forall \boldsymbol{b} \in 2^{\leq \omega}\right)$ (if $f_{X}(x) \leq \boldsymbol{b}$, then $\left.\boldsymbol{b} \in S\right)$. And since $S$ is $\mathcal{U}$-convergent, it suffices to show that $\left(\forall b \in 2^{<\omega}\right)$ (if $f_{X}(x) \leq b$, then $\left.b \in S\right)$. So choose $b \in 2^{<\omega}$ such that $f_{X}(x) \leq b$. By Lemma 7.7, we have $C l\left(X_{f_{X}(x)}\right) \subseteq C l\left(X_{b}\right)$, in which case $X_{f_{X}(x)} \subseteq C l\left(X_{b}\right)$. Also, $x \in X_{f_{X}(x)}$, by definition of $f_{X}$. So $x \in C l\left(X_{b}\right)$. So there is some $y \in \mathbf{B}(x, r) \cap X_{b} \subseteq$ $h_{f_{X}}(S) \cap X_{b}$. Since $y \in X_{b}$, we have $f_{X}(y)=b$. And since $y \in h_{f_{X}}(S)$, we have $f_{X}(y) \in S$. So $b \in S$, as desired.

(Case 2) $f_{X}(x) \in 2^{\omega}$. Remark: It was for this case that we added the $\varepsilon$-clause, that is, Clause (4), to Lemma 7.1. See Remarks 7.2 and 7.5. Recall that $x \in X_{f_{X}(x)}=$ $\bigcap_{n \in \mathbb{N}} O_{\left.f_{X}(x)\right|_{n}}$. Choose $n \in \mathbb{N}$ such that $1 /(n+1)<r / 2$. Note that $\left.f_{X}(x)\right|_{n} \leq f_{X}(x)$, so that $\left.f_{X}(x) \in \llbracket f_{X}(x)\right|_{n} \rrbracket$. So to show that $f_{X}(x) \in \operatorname{Int}_{2} \leq \omega(S)$, it suffices to show that $\left.\llbracket f_{X}(x)\right|_{n} \rrbracket \subseteq S$. That is, it suffices to show that $\left(\forall \boldsymbol{b} \in 2^{\leq \omega}\right)$ (if $\left.f_{X}(x)\right|_{n} \leq \boldsymbol{b}$, then $\boldsymbol{b} \in S$ ). And since $S$ is $\mathcal{U}$-convergent, it suffices to show that $\left(\forall b \in 2^{<\omega}\right)$ (if $\left.f_{X}(x)\right|_{n} \leq b$, then $b \in S)$. So choose $b \in 2^{<\omega}$ such that $\left.f_{X}(x)\right|_{n} \leq b$.

Clearly, $x \in O_{\left.f_{X}(x)\right|_{n}}$. So $d\left(x, X_{\left.f_{X}(x)\right|_{n}}\right) \leq 1 /(n+1)$, by the definition of $X_{\left.f_{X}(x)\right|_{n}}$ as

$$
X_{\left.f_{X}(x)\right|_{n}}={ }_{\mathrm{df}} M\left(O_{\left.f_{X}(x)\right|_{n}}, \frac{1}{\left|f_{X}(x)\right|_{n} \mid+1}\right)=M\left(O_{\left.f_{X}(x)\right|_{n}}, \frac{1}{n+1}\right) .
$$

So $d\left(x, X_{\left.f_{X}(x)\right|_{n}}\right)<r / 2$. Choose $y \in X_{\left.f_{X}(x)\right|_{n}}$ with $d(x, y)<r / 2$. By Lemma 7.7, $X_{\left.f_{X}(x)\right|_{n}} \subseteq C l\left(X_{b}\right)$. So $y \in C l\left(X_{b}\right)$. Choose any $z \in X_{b}$ with $d(y, z)<r / 2$. So $d(x, z)<r$. So $z \in \mathbf{B}(x, r)$ and $f_{X}(z)=b$. So $z \in h_{f_{X}}(S)$ and $f_{X}(z)=b$. So $f_{X}(z) \in S$ and $f_{X}(z)=b$. So $b \in S$, as desired.

Given Lemma 6.4, we have an embedding $h_{\mathcal{U}}$ from $\mathcal{I}\left(2^{<\omega}\right)$ into $\mathcal{J}_{\mathcal{U}}$. And given Lemma 9.1, we have an embedding $h^{*}$ from $\mathcal{J}_{\mathcal{U}}$ into $\mathcal{I}(X)$. Thus, $h^{*} \circ h_{\mathcal{U}}$ is an embedding from $\mathcal{I}\left(2^{<\omega}\right)$ into $\mathcal{I}(X)$. This is what was promised in Lemma 5.7 and suffices for Theorem 5.5 and hence for our main result, Theorem 2.2.

\$10. A proof of the decomposition lemma. The proof here of Lemma 7.1 is adapted from the proof in Rasiowa \& Sikorski (1963) of Theorem III, 7.1, itself derived from Tarski (1938), and McKinsey \& Tarski (1944). We had to add minor considerations in order to ensure the $\varepsilon$-clause. Before we launch into the proof, some preliminary definitions and lemmas. A subset $S$ of a topological space $X$ is nowhere dense iff $\operatorname{Int}(\mathrm{Cl}(S))=\emptyset$. If $\varepsilon>0$, then a subset $S$ of a metric space $X$ is an $\varepsilon$-set iff $d(x, y) \geq \varepsilon$ for any pair of distinct points $x, y \in S$. If $S \subseteq S^{\prime} \subseteq X$, where $X$ is a metric space, then $S$ is a maximal $\varepsilon$-subset of $S^{\prime}$ if $S$ is not a proper subset of any other $\varepsilon$-set contained in $S^{\prime}$. Our first two lemmas concern nowhere dense sets and $\varepsilon$-sets.

LEMMA 10.1.

1. The union of finitely many nowhere dense sets is nowhere dense.

2. If $O$ is open and $S$ is nowhere dense, then $C l(O)=C l(O-S)$.

Proof. (1) It suffices to show that the union of two nowhere dense sets is nowhere dense. It can easily be checked that, for any two sets $S_{1}$ and $S_{2}, \operatorname{Int}\left(S_{1} \cup S_{2}\right) \subseteq \operatorname{Int}\left(S_{1}\right) \cup \operatorname{Cl}\left(S_{2}\right)$. Now suppose that $S_{1}$ and $S_{2}$ are nowhere dense, that is, $\operatorname{Int}\left(\operatorname{Cl}\left(S_{1}\right)\right)=\operatorname{Int}\left(C l\left(S_{2}\right)\right)=\emptyset$. Note: $\operatorname{Int}\left(C l\left(S_{1} \cup S_{2}\right)\right)=\operatorname{Int}\left(C l\left(S_{1}\right) \cup C l\left(S_{2}\right)\right) \subseteq \operatorname{Int}\left(C l\left(S_{1}\right)\right) \cup \operatorname{Cl}\left(C l\left(S_{2}\right)\right) \subseteq \emptyset \cup$ $C l\left(S_{2}\right) \subseteq C l\left(S_{2}\right)$. Thus, $\operatorname{Int}\left(C l\left(S_{1} \cup S_{2}\right)\right) \subseteq \operatorname{Int}\left(C l\left(S_{2}\right)\right)=\emptyset$. 
(2) Suppose that $O$ is open and $S$ is nowhere dense. Clearly, $\mathrm{Cl}(\mathrm{O}-\mathrm{S}) \subseteq \mathrm{Cl}(\mathrm{O})$. So it suffices to show that $C l(O) \subseteq C l(O-S)$. Suppose that $x \in C l(O)-C l(O-S)$. Since $x \notin C l(O-S)$, there is an open set $O^{\prime}$ with $x \in O^{\prime}$ and with $O^{\prime} \cap(O-S)=\emptyset$. So $\left(O^{\prime} \cap O\right)-S=\emptyset$. So $\left(O^{\prime} \cap O\right) \subseteq S$. Also, since $x \in O^{\prime}$ and $x \in C l(O)$, the open set $\left(O^{\prime} \cap O\right)$ is nonempty. So $\mathrm{Cl}(S)$ has a nonempty interior, contradicting $S$ being nowhere dense.

LEMma 10.2. (Rasiowa \& Sikorski, 1963, pp. 106-107).

\section{Every $\varepsilon$-set is closed.}

2. Every $\varepsilon$-set in a dense-in-itself metric space is nowhere dense.

3. Every subset of a metric space has a maximal $\varepsilon$-subset.

4. If $S$ is a maximal $\varepsilon$-subset of $S^{\prime}$, then $\left(\forall x \in C l\left(S^{\prime}\right)\right)(d(x, S) \leq \varepsilon)$.

5. If $O$ is an open subset of a dense-in-itself metric space $X$ and $\varepsilon>0$, then there are disjoint closed nowhere dense sets $S_{1}, S_{2}, S_{3} \subseteq O$ such that $(\forall x \in C l(O))$ $\left(d\left(x, S_{i}\right) \leq \varepsilon\right)$.

Proof. (1) Suppose that $S$ is an $\varepsilon$-set in a metric space $X$, and that $x \in C l(S)$. Then $\mathbf{B}(x, \varepsilon / 2) \cap S$ is nonempty. Note that $(\forall y, z \in \mathbf{B}(x, \varepsilon / 2))$ (if $y \neq z$, then $d(y, z)<\varepsilon / 2)$ and $(\forall y, z \in S)$ (if $y \neq z$, then $d(y, z) \geq \varepsilon)$. So $\mathbf{B}(x, \varepsilon / 2) \cap S$ has at most one member, say $y$. Note that $y=x$ : otherwise $\mathbf{B}(x, d(x, y) / 2)$ would be an open set containing $x$ and disjoint from $S$, which contradicts $x \in C l(S)$. So $x \in S$.

(2) Suppose that $S$ is an $\varepsilon$-set in a dense-in-itself metric space $X$. Since $S$ is closed, it suffices to show that $\operatorname{Int}(S)$ is empty. Suppose that $x \in \operatorname{Int}(S)$. Then $x \in \mathbf{B}(x, \varepsilon / 2) \subseteq$ $S$. Since $X$ is dense-in-itself, $\{x\}$ is not open, in which case $(\exists y \in \mathbf{B}(x, \varepsilon / 2))(y \neq x)$. Note that $d(x, y)<\varepsilon / 2$, since $y \in \mathbf{B}(x, \varepsilon / 2))$. On the other hand $y \in S$, since $y \in$ $\mathbf{B}(x, \varepsilon / 2)) \subseteq S$ : therefore, $d(x, y) \geq \varepsilon$, since $S$ is an $\varepsilon$-set and $x \in S$. A contradiction.

(3) Suppose that $S$ is a subset of a metric space $X$. Let $\mathcal{E}$ be the class of $\varepsilon$-subsets of $S$. Note that $\mathcal{E}$ is nonempty, since $\emptyset \in \mathcal{E}$. Also, the union of any increasing chain (ordered by set inclusion) of members of $\mathcal{E}$ is a member of $\mathcal{E}$. So $\mathcal{E}$ has a maximal member.

(4) Suppose that $S$ is a maximal $\varepsilon$-subset of $S^{\prime}$, and $x \in C l\left(S^{\prime}\right)$ but $(d(x, S)>\varepsilon)$. $S^{\prime}$ is nonempty, since $x \in C l\left(S^{\prime}\right)$. So $S$ is also nonempty: this follows from the fact that any singleton subset of $S^{\prime}$ is an $\varepsilon$-subset of $S^{\prime}$, so that any maximal $\varepsilon$-subset of $S^{\prime}$ is nonempty. So $d(x, S)$ is finite. Let $\delta=d(x, S)-\varepsilon$. Since $x \in C l\left(S^{\prime}\right)$, there is a point $y \in \mathbf{B}(x, \delta) \cap S^{\prime}$. Now, $d(x, S) \leq d(y, S)+d(x, y)<d(y, S)+\delta=d(y, S)+d(x, S)-\varepsilon$. So $d(y, S)>\varepsilon$. So $y \notin S$, and $S \cup\{y\}$ is an $\varepsilon$-subset of $S^{\prime}$. But this contradicts the maximality of the $\varepsilon$-subset $S$.

(5) Suppose that $O$ is an open subset of a dense-in-itself metric space $X$ and that $\varepsilon>0$. Let $S_{1}$ be a maximal $\varepsilon$-subset of $O, S_{2}$ a maximal $\varepsilon$-subset of $O-S_{1}$, and $S_{3}$ a maximal $\varepsilon$-subset of $O-\left(S_{1} \cup S_{2}\right) . S_{1}, S_{2}$, and $S_{3}$ are clearly disjoint. By (1) and (2), they are closed and nowhere dense. By Lemma 10.1, $C l(O)=C l\left(O-S_{1}\right)=C l\left(O-\left(S_{1} \cup S_{2}\right)\right)$. So, by (4), $(\forall x \in C l(O))\left(d\left(x, S_{i}\right) \leq \varepsilon\right)$, for $i=1,2$, or 3 .

LEMmA 10.3. (A separation lemma, simplifying Rasiowa \& Sikorski (1963, p. 105, 6.1)). Suppose that $C_{1}$ and $C_{2}$ are disjoint closed subsets of a metric space $X=\langle X, d\rangle$. Then there is an open set $O$ such that $C_{1} \subseteq O$ and $C l(O) \cap C_{2}=\emptyset$.

Proof. It suffices to show this in the case where $C_{1}$ and $C_{2}$ are nonempty. Let $O=$ $\left\{x \in X: d\left(x, C_{1}\right)<d\left(x, C_{2}\right) / 2\right\}$. To see that $C_{1} \subseteq O$, suppose that $x \in C_{1}$. Then 
$d\left(x, C_{1}\right)=0$. On the other hand, since $C_{2}$ is closed and $x \notin C_{2}, d\left(x, C_{2}\right)>0$. So $x \in O$ as desired.

To see that $C l(O) \cap C_{2}=\emptyset$, suppose that $x \in C l(O) \cap C_{2}$. Note that $x \notin C_{1}$, since $x \in C_{2}$. So, since $C_{1}$ is closed and $x \notin C_{1}, d\left(x, C_{1}\right)>0$. Let $\delta=d\left(x, C_{1}\right) / 3$. Since $x \in C l(O)$, there is some $y \in O \cap \mathbf{B}(x, \delta)$. So $d(x, y)<\delta$. Note:

$d\left(y, C_{2}\right) \leq d\left(x, C_{2}\right)+d(x, y)<d\left(x, C_{2}\right)+\delta$.

So $d\left(x, C_{2}\right)>d\left(y, C_{2}\right)-\delta$.

Also, $d\left(y, C_{2}\right)>2\left(d\left(y, C_{1}\right)\right)$, since $y \in O$.

So $d\left(x, C_{2}\right)>2\left(d\left(y, C_{1}\right)\right)-\delta$.

Also, $d\left(x, C_{1}\right) \leq d\left(y, C_{1}\right)+d(x, y)<d\left(y, C_{1}\right)+\delta$.

So $2\left(d\left(y, C_{1}\right)\right)>2\left(d\left(x, C_{1}\right)\right)-2 \delta$.

So $d\left(x, C_{2}\right)>2\left(d\left(x, C_{1}\right)\right)-3 \delta=d\left(x, C_{1}\right)>0$.

So $x \notin C_{2}$, since $C_{2}$ is closed. A contradiction.

Finally, to see that $O$ is open, let $x \in O$. And let $\varepsilon=\left(d\left(x, C_{2}\right)-2\left(d\left(x, C_{1}\right)\right)\right) / 3$. Note that $\varepsilon>0$, since $d\left(x, C_{1}\right)<d\left(x, C_{2}\right) / 2$. It will suffice to show that $\mathbf{B}(x, \varepsilon) \subseteq O$. So, choose $y \in \mathbf{B}(x, \varepsilon)$. Note:

$d\left(x, C_{2}\right) \leq d\left(y, C_{2}\right)+d(x, y)<d\left(y, C_{2}\right)+\varepsilon$.

So $d\left(y, C_{2}\right)>d\left(x, C_{2}\right)-\varepsilon$.

Also $d\left(y, C_{1}\right) \leq d\left(x, C_{1}\right)+d(x, y)<d\left(x, C_{1}\right)+\varepsilon$.

So $d\left(y, C_{2}\right)-2\left(d\left(y, C_{1}\right)\right)>\left(d\left(x, C_{2}\right)-\varepsilon\right)-2\left(d\left(x, C_{1}\right)+\varepsilon\right)=\left(d\left(x, C_{2}\right)-\right.$ $\left.2\left(d\left(x, C_{1}\right)\right)\right)-3 \varepsilon=0$.

So $d\left(y, C_{2}\right)>2\left(d\left(y, C_{1}\right)\right)$.

So $y \in O$, as desired.

COROLlary 10.4. (A separation corollary). Suppose that $C_{1}, C_{2}$ and $C_{3}$ are disjoint closed subsets of a metric space $X=\langle X, d\rangle$. Then, there are open sets $O_{1}$ and $O_{2}$ such that $C_{1} \subseteq O_{1}, C_{2} \subseteq O_{2}$ and such that $\mathrm{Cl}\left(O_{1}\right), C l\left(O_{2}\right)$ and $C_{3}$ are disjoint.

Proof. Suppose that $C_{1}, C_{2}$ and $C_{3}$ are disjoint closed subsets of a metric space $X=$ $\langle X, d\rangle$. Note that $C_{1}$ and $C_{2} \cup C_{3}$ are disjoint closed subsets of $X$. So, by Lemma 10.3, there is an open set $O_{1}$ such that $C_{1} \subseteq O_{1}$ and $C l\left(O_{1}\right)$ is disjoint from $C_{2} \cup C_{3}$. Note that $C_{2}$ and $\mathrm{Cl}\left(\mathrm{O}_{1}\right) \cup C_{3}$ are disjoint closed subsets of $X$. So, again by Lemma 10.3 , there is an open set $O_{2}$ such that $C_{2} \subseteq O_{2}$ and $C l\left(O_{2}\right)$ is disjoint from $\operatorname{Cl}\left(O_{1}\right) \cup C_{3}$. Note that $\mathrm{Cl}\left(\mathrm{O}_{1}\right), \mathrm{Cl}\left(\mathrm{O}_{2}\right)$ and $\mathrm{C}_{3}$ are disjoint, as desired.

Proof of Lemma 7.1. Suppose that $X$ is a dense-in-itself metric space, that $O \subseteq X$ is nonempty and open and that $\varepsilon>0$. By induction on $n \in \mathbb{N}$, we will define, for each $n \in \mathbb{N}$, three disjoint subsets of $O: L_{n}$ ( $L$ for left), $R_{n}$ ( $R$ for right), $M_{n}$ ( $M$ for middle), so that for each $n \in \mathbb{N}$,

1. $L_{n}$ and $R_{n}$ are open;

2. $M_{n}$ is closed and nowhere dense;

3. the following sets are disjoint: $X-O, C l\left(L_{n}\right), C l\left(R_{n}\right), M_{n}$;

4. $(\forall m<n)\left(C l\left(L_{m}\right) \subseteq L_{n} \& C l\left(R_{m}\right) \subseteq R_{n} \& M_{m} \subseteq M_{n}\right)$;

5. if $n>0$, then $\left(\forall x \in C l(O)-\left(L_{n} \cup R_{n}\right)\right)\left(d\left(x, L_{n}\right) \leq \varepsilon / n \& d\left(x, R_{n}\right) \leq\right.$ $\left.\varepsilon / n \& d\left(x, M_{n}\right) \leq \varepsilon / n\right)$

6. if $n>0$, then $L_{n}, R_{n}$, and $M_{n}$ are nonempty. 
After defining the $L_{n}, R_{n}$, and $M_{n}$, we will define $L(O, \varepsilon), R(O, \varepsilon)$, and $M(O, \varepsilon)$, and show that these three sets have the properties in the statement of the lemma we are proving.

Let $L_{0}=R_{0}=M_{0}=\emptyset$. Suppose that disjoint $L_{n}, R_{n}$, and $M_{n}$ have been defined so that (1)-(6) hold. By Lemma 10.2(5), there are disjoint closed nowhere dense subsets $L_{n}^{\prime}$, $R_{n}^{\prime}$, and $M_{n}^{\prime}$ of the open set $O_{n}=O-\left(C l\left(L_{n}\right) \cup C l\left(R_{n}\right) \cup M_{n}\right)$, such that, if $Y=L_{n}^{\prime}, R_{n}^{\prime}$, or $M_{n}^{\prime}$,

$$
\left(\forall x \in C l\left(O_{n}\right)\right)(d(x, Y) \leq \varepsilon /(1+n)) .
$$

Let $M_{n+1}=M_{n} \cup M_{n}^{\prime}$. Note that the following three sets are closed and disjoint: $C l\left(L_{n}\right) \cup L_{n}^{\prime}, C l\left(R_{n}\right) \cup R_{n}^{\prime}, M_{n+1} \cup(X-O)$. So, by Corollary 10.4, there are open sets $L_{n+1}$ and $R_{n+1}$ such that $C l\left(L_{n}\right) \cup L_{n}^{\prime} \subseteq L_{n+1}$ and $C l\left(R_{n}\right) \cup R_{n}^{\prime} \subseteq R_{n+1}$ and the following three sets are disjoint: $C l\left(L_{n+1}\right), C l\left(L_{n+1}\right), M_{n+1} \cup(X-O)$. So the following four sets are disjoint: $C l\left(L_{n+1}\right), C l\left(L_{n+1}\right), M_{n+1},(X-O)$. Note that (1)-(3) hold for $n+1$. Also, given the inductive hypothesis that (4) holds for $n$, it is easy to see that (4) also holds for $n+1$, since $C l\left(L_{n}\right) \subseteq L_{n+1}, C l\left(R_{n}\right) \subseteq R_{n+1}$, and $M_{n} \subseteq M_{n+1}$.

To show that (5) holds for $n+1$, it suffices, given $(*)$, to show that $C l(O)-\left(L_{n+1} \cup\right.$ $\left.R_{n+1}\right) \subseteq C l\left(O_{n}\right)$. Recall that $O_{n}=O-\left(C l\left(L_{n}\right) \cup C l\left(R_{n}\right) \cup M_{n}\right)=\left(O-\left(C l\left(L_{n}\right) \cup\right.\right.$ $\left.C l\left(R_{n}\right)\right)-M_{n}$. So, by Lemma $10.1(2), C l\left(O_{n}\right)=C l\left(O-\left(C l\left(L_{n}\right) \cup C l\left(R_{n}\right)\right)\right)$. So it suffices to show that $C l(O)-\left(L_{n+1} \cup R_{n+1}\right) \subseteq C l\left(O-\left(C l\left(L_{n}\right) \cup C l\left(R_{n}\right)\right)\right)$. Here goes:

$$
\begin{aligned}
& C l(O)-\left(L_{n+1} \cup R_{n+1}\right) \\
& \quad \subseteq C l(O)-\left(C l\left(L_{n}\right) \cup C l\left(R_{n}\right)\right), \text { since } C l\left(L_{n}\right) \subseteq L_{n+1} \text { and } C l\left(R_{n}\right) \subseteq R_{n+1}, \\
& \quad \subseteq C l(O)-C l\left(L_{n} \cup R_{n}\right) \\
& \quad \subseteq C l\left(O-C l\left(L_{n} \cup R_{n}\right)\right) \\
& \quad \subseteq C l\left(O-C l\left(L_{n}\right) \cup C l\left(R_{n}\right)\right) .
\end{aligned}
$$

The step marked (\#) is justified because for any two subsets $S_{1}$ and $S_{2}$ of a topological space, $C l\left(S_{1}\right)-C l\left(S_{2}\right) \subseteq C l\left(S_{1}-C l\left(S_{2}\right)\right)$. This can easily be checked.

To show that (6) holds for $n+1$, it suffices to note that $L_{1}, R_{1}$, and $M_{1}$ are nonempty: this follows from the fact that $L_{1}^{\prime}, R_{1}^{\prime}$, and $M_{1}^{\prime}$ are nonempty, which follows from their definition.

Now define

$$
\begin{aligned}
L(O, \varepsilon) & ={ }_{\mathrm{df}} \bigcup_{n} L_{n} \\
R(O, \varepsilon) & ={ }_{\mathrm{df}} \bigcup_{n} R_{n} \\
M(O, \varepsilon) & =\mathrm{df}_{\mathrm{df}} O-(L(O, \varepsilon) \cup R(O, \varepsilon)) .
\end{aligned}
$$

Note that $\bigcup_{n} M_{n} \subseteq M(O, \varepsilon)$.

The sets $L(O, \varepsilon), R(O, \varepsilon)$, and $M(O, \varepsilon)$ are nonempty: this follows from the fact that $L_{1}^{\prime} \subseteq L(O, \varepsilon), R_{1}^{\prime} \subseteq R(O, \varepsilon)$, and $M_{1}^{\prime} \subseteq M(O, \varepsilon)$. It is also obvious that $L(O, \varepsilon), R(O, \varepsilon)$, and $M(O, \varepsilon)$ are pairwise disjoint, and that $O=L(O, \varepsilon) \cup \dot{\cup} R(O, \varepsilon) \dot{\cup} M(O, \varepsilon)$ : this is Clause (1) of the lemma. As for Clause (2) of the lemma, it suffices to note that each of $L(O, \varepsilon)$ and $R(O, \varepsilon)$ is the union of open sets.

We now turn to Clause (4) of the lemma, the $\varepsilon$-clause. By (*) above,

$$
\left(\forall x \in C l\left(O_{0}\right)\right)\left(d\left(x, M_{0}^{\prime}\right) \leq \varepsilon\right) .
$$

But $O_{0}=O$, and $M_{0}^{\prime}=M_{1} \subseteq M(O, \varepsilon)$. So $(\forall x \in O)(d(x, M(O, \varepsilon)) \leq \varepsilon)$, as desired.

We finally turn to Clause (3) of the lemma. Let $C$ be the following closed set: $\mathrm{Cl}(\mathrm{O})-$ $(L(O, \varepsilon) \cup R(O, \varepsilon))$. It follows from (5), above, that

$$
(\forall x \in C)(d(x, L(O, \varepsilon))=d(x, R(O, \varepsilon))=d(x, M(O, \varepsilon))=0) .
$$


So $C \subseteq C l(L(O, \varepsilon))$. Indeed, since $C$ is disjoint from $L(O, \varepsilon)$, we have $C \subseteq C l(L(O, \varepsilon))-$ $L(O, \varepsilon)=\partial(L(O, \varepsilon))$. On the other hand, $L(O, \varepsilon) \subseteq C l(O)-R(O, \varepsilon)$. Since the righthand side of this inclusion is closed, $C l(L(O, \varepsilon)) \subseteq C l(O)-R(O, \varepsilon)$. So $\partial(L(O, \varepsilon))=$ $C l(L(O, \varepsilon))-L(O, \varepsilon) \subseteq C$. So $\partial(L(O, \varepsilon))=C$. Similarly, $\partial(R(O, \varepsilon))=C$. Thus,

$$
\partial(L(O, \varepsilon))=\partial(R(O, \varepsilon))=C l(O)-(L(O, \varepsilon) \cup R(O, \varepsilon)) .
$$

As for $C l(M(O, \varepsilon))$, it follows from ( $\dagger)$ that $C \subseteq C l(M(O, \varepsilon))$. Also, by the definition of $M(O, \varepsilon)$, we have $M(O, \varepsilon) \subseteq C$. So, since $C$ is closed, we have $C l(M(O, \varepsilon)) \subseteq C$. Thus $C l(M(O, \varepsilon))=C=C l(O)-(L(O, \varepsilon) \cup R(O, \varepsilon))$.

§11. Acknowledgments. Thanks to the audience at the Ninth International Tbilisi Symposium on Language, Logic and Computation (2011) in Kutaisi, Georgia, for listening to me present an early version of this paper. Special thanks to each of David Gabelaia, Nick Bezhanishvili, Roman Kontchakov, and Mamuka Jibladze for indulging me by letting me explain the proof in detail in the case $X=\mathbb{R}$. And special thanks to three very helpful anonymous referees, who worked through two earlier drafts of this paper and provided invaluable suggestions and comments.

\section{BIBLIOGRAPHY}

Aiello, M., van Benthem, J., \& Bezhanishvili, G. (2003). Reasoning about space: The modal way. Journal of Logic and Computation, 13, 889-920.

Bezhanishvili, G., \& Gehrke, M. (2005). Completeness of S4 with respect to the real line: Revisited. Annals of Pure and Applied Logic, 131, 287-301.

Blok, W. (1976). Varieties of Interior Algebras. Dissertation, University of Amsterdam.

Dugundji, J. (1966). Topology. Boston, MA: Allyn and Bacon.

Engelking, R. (1989). General Topology. Berlin, Germany: Heldermann Verlag.

Fine, K. (1974). An ascending chain of S4 logics. Theoria, 40, 110-116.

Gierz, G., Hoffmann, K. H., Keimel, K., Lawson, J. D., Mislove, M., \& Scott, D. S. (2003). Continuous Lattices and Domains. Cambridge, UK: Cambridge University Press.

Goldblatt, R. (1980). Diodorean modality in Minkowski spacetime. Studia Logica, 39, 219-236.

Hodkinson, I. (2012). Simple completeness proofs for some spatial logics of the real line. Available from: http://www.doc.ic.ac.uk/ imh/papers/boxoverR.pdf.

Kremer, P. (forthcoming). The incompleteness of $\mathrm{S} 4 \oplus \mathrm{S} 4$ for the product space $\mathbb{R} \times \mathbb{R}$. Studia Logica. A preprint of this paper is available here: http://www.individual.utoronto. ca/philipkremer/onlinepapers/RxR.pdf.

Kremer, P., \& Mints, G. (2005). Dynamic topological logic. Annals of Pure and Applied Logic, 131, 133-158.

Kuratowski, C. (1922). Sur l'opération $\bar{A}$ de l'Analysis Situs. Fundamenta Mathematicae, 3, 182-199.

Lando, T. (2012). Completeness of S4 for the Lebesgue measure algebra based on the unit interval. Journal of Philosophical Logic, 41, 287-316.

Lando, T., \& Sarenac, D. (2011) Fractal Completeness Techniques in Topological Modal Logic: Koch Curve, Limit Tree, and the Real Line. Available from: http://philosophy. berkeley.edu/file/698/FractalCompletenessTechniques.pdf.

Makinson, D. (1966). On some completeness theorems in modal logic. Zeitschrift fr mathematische Logik und Grundlagen der Mathematik, 12, 379-384. 
McKinsey, J. C. C. (1941). A solution of the decision problem for the Lewis systems S2 and S4, with an application to topology. The Journal of Symbolic Logic, 6, 117-134.

McKinsey, J. C. C., \& Tarski, A. (1944). The algebra of topology. Annals of Mathematics, 45, 141-191.

Mints, G., \& Zhang, T. (2005). A proof of topological completeness for S4 in (0, 1). Annals of Pure and Applied Logic, 133, 231-245.

Rasiowa, H., \& Sikorski, R. (1963). The Mathematics of Metamathematics. Państowowe Wydawnictwo Naukowe, Warsaw.

Stone, M. (1936). The theory of representations of Boolean algebras. Transactions of the American Mathematical Society, 40, 37-111.

Tarski, A. (1938). Der Ausgangkalkül und die Topologie. Fundamenta Mathematicae, 31, 103-134.

van Benthem, J., Bezhanishvili, G., ten Cate, B., \& Sarenac, D. (2006). Multimodal logics of products of topologies. Studia Logica, 84, 369-392.

Zakharyaschev, M., Wolter, F., \& Chagrov, A. (1997). Advanced modal logic. In Gabbay, D., and Guenther, F., editors. Handbook of Philosophical Logic(second edition), Vol. 3. Dordrecht, The Netherlands: Kluwer Academic Publishers, pp. 83-266.

\author{
DEPARTMENT OF PHILOSOPHY \\ UNIVERSITY OF TORONTO \\ 170 ST. GEORGE STREET \\ TORONTO ON, CANADA, M5R 2M8
}

E-mail: kremer@utsc.utoronto.ca 